\title{
Adaptive neural network tracking control for underactuated systems with matched and mismatched disturbances
}

\author{
Pengcheng Liu $\mathbb{( \mathbb { D } )}$ - Hongnian Yu(i) \\ Shuang Cang
}

Received: 27 April 2018 / Accepted: 27 July 2019 / Published online: 8 October 2019

(C) The Author(s) 2019

\begin{abstract}
This paper studies neural network-based tracking control of underactuated systems with unknown parameters and with matched and mismatched disturbances. Novel adaptive control schemes are proposed with the utilization of multi-layer neural networks, adaptive control and variable structure strategies to cope with the uncertainties containing approximation errors, unknown base parameters and time-varying matched and mismatched external disturbances. Novel auxiliary control variables are designed to establish the controllability of the non-collocated subset of the underactuated systems. The approximation errors and the matched and mismatched external disturbances are efficiently counteracted by appropriate design of robust compensators. Stability and convergence of the time-varying reference trajectory are shown in the sense of Lyapunov. The parameter updating laws for the designed control schemes are derived using the projection approach to reduce the tracking error as small as desired. Unknown dynamics of the
\end{abstract}

P. Liu

Cardiff School of Technologies, Cardiff Metropolitan

University, Cardiff CF5 2YB, UK

H. Yu ( $₫)$

School of Engineering and the Built Environment,

Edinburgh Napier University, 10 Colinton Road,

Edinburgh EH10 5DT, UK

e-mail: h.yu@napier.ac.uk

S. Cang

School of Economics and Management, Yanshan University, Qinhuangdao 066004, China non-collocated subset is approximated through neural networks within a local region. Finally, simulation studies on an underactuated manipulator and an underactuated vibro-driven system are conducted to verify the effectiveness of the proposed control schemes.

Keywords Adaptive tracking control .

RBF neural networks .

Underactuated systems · Auxiliary control variables . Matched and mismatched disturbances

\section{Introduction}

Underactuated mechanical systems (UMSs) are rapidly growing research fields that combine control and robotics societies [1-8]. They have extensive applications such as UAVs, underground vehicles, spacecraft, humanoid robots and vibro-driven robots [9-12]. UMSs have more degrees-of-freedom (DOF) $n$ than independent control inputs $m$; thus, $(n-m)$ DOF are not directly controllable, which characterize the nature of underactuation. This nature is made possible for the UMSs to undertake complicated tasks with a reduced number of actuators that in turn implies the reduction in weight and energy consumption. Challenge that faced by control of underactuated systems is the existence of underactuation and some undesirable properties such as being in a non-minimum phase and/or possessing an undetermined relative degree, which makes conventional approaches not directly applica- 
ble, particularly for the issue of trajectory tracking control. Design of the control schemes for UMSs is intractable because of the internal dynamics and non-holonomic property, and they are not feedback linearizable [13]. Besides, the uncertainties in system model, as well as the matched and mismatched disturbances, make control of UMSs more challenging.

The complexity of control problem related to UMSs can be reduced when the objective is merely to stabilize a subset of the UMSs' DOF. In the literature, a great number of existing control system designs for UMSs explore the concept of linearization through partial feedback [14-18]. Linear systems can be utilized to capture the underactuated dynamics within a local range; however, global stabilization of the underactuated dynamics is still unavailable under this approach. Other prevailing techniques such as inverse dynamics [19,20], sliding mode/variable structure [14, $21,22]$, energy/passivity-based approaches [17,23,24] have been extensively exploited. Furthermore, practical requirements are raised from the current applications, in which the adaptability of UMSs is extremely crucial when facing environments with uncertainties. For instance, microrobotic systems work across vulnerable media in restricted space for minimally invasive sensing and risk intervention in pipeline inspection, endoscopic assistance, underwater exploration, etc. However, an exact dynamic model is intractable to obtain due to the presence of frictions, unknown disturbances, time-varying parameters, etc. As a result, adaptive control schemes for generic UMSs have attracted great attentions. Considering uncertainties and ocean disturbances, a control system using leader-follower formation was studied in [25] for underactuated autonomous surface vehicles. Dynamic surface control technique and neural networks (NNs) were used to construct the control scheme. A hierarchical sliding mode control system with adaptive and fuzzy inclusions was studied for uncertain UMSs in [14], where different layers of sliding surface are constructed to cope with the uncertainties and disturbances and fuzzy models are designed to approximate the nonlinearities.

It is evident that description of dynamic couplings between the actuated and passive subsystems of UMSs is typically highly nonlinear. Therefore, it is plausible to consider the employment of approximation approaches to map the coupling between the torques applied at the actuated subsystem and the resulting accelerations of the passive subsystem, with the intent of achieving control globally. As such, in this paper, nonlinear control approach is investigated by employing multi-layer NNs. NNs have versatile features such as learning capability mapping and parallel processing. An attractive feature of NNs is that their synaptic weights are online updated without any offline learning phases. NNs have the property of robustness; thus, they have been widely applied in various robotic systems to address the stabilization problem [26-28]. The issue of tracking control of UMSs based on NNs has attracted extensive attentions. Optimal motion control using NNs and stochastic adaptive concepts was studied towards the Pendubot in [29] and a WIP system in [30]. For UMSs with full-state constraints containing a Moore-Penrose inverse term, an adaptive NN control system was proposed in [31]. In [31], the authors developed two decentralized output feedback control systems based on adaptive NN to tackle with immeasurable states and unknown time delays in UMSs. Towards a wheeled mobile robot that is non-holonomic with unknown parameters and uncertain dynamics, an adaptive tracking control scheme was presented in [32] to tune the kinematic controller gain online and minimize the tracking error in velocity. A bio-inspired tracking control scheme based on NNs was developed in [33] for an underactuated surface vessel with unknown system dynamics. A cart-pendulum system with unknown dynamics was studied in [34], and a trajectory tracking control scheme of the pendulum subsystem based on adaptive $\mathrm{NN}$ was designed instead of considering the position of the cart. In [35], an output feedback control system based on NNs was proposed for tracking control of a spherical inverted pendulum. A combined PID and neural network compensation approach was proposed in [36] to control a wheel-driven mobile pendulum system, and the results were experimentally analysed. From the literature, it is noted that relatively few studies have addressed the issue of tracking control for UMSs, particularly when the disturbance exists in the non-collocated subsystem, which is mismatched with the control actions. Also, it is noted that very few reported studies towards this topic have presented rigorous analysis of trajectories of the closedloop system for UMSs. Therefore, trajectory tracking control for UMSs with uncertainties and disturbances is still an open problem and requires in-depth investigations. 
Through the utilization of the unique physical properties of the UMSs, the overall underactuated system breaks down into two subsystems, i.e. a fully actuated subsystem and an unactuated (passive) subsystem. Radial basis neural network (RBFNN) has simple structure and fast convergence rate, and it can overcome the local minimum problem; therefore, it is utilized as a nonlinear function approximator of uncertain dynamics of the unactuated (passive) subsystem of the UMSs. The NN control has the ability of universal approximation, and it has been thoroughly studied on discrete-time system [37-40] and continuous-time systems [41-44]. There are very limited studies using $\mathrm{NN}$ to approximate the system dynamics of UMSs. In this paper, we develop NNbased adaptive tracking control schemes to cope with the internal uncertain dynamics and external disturbances, and auxiliary control variables are explicitly designed to close the unactuated feedback loops. RBFNN is adopted to approximate the mismatched system uncertainties, and the adaptive control algorithm is constructed to estimate the NNs approximation error and the bounded mismatched disturbance. The combination of NN approximation, variable structure control and adaptive approach makes the constructed new controller more robust, and as such, errors resulting from trajectory tracking, parameter uncertainties, mismatched external disturbances and NN approximation are counteracted. Theoretical background of these methods is presented with rigorous analysis and developed in detail for some examples. The schemes promote the utilization of linear filters in the control input such that the system robustness is improved. Stability of the system dynamics and convergence of the time-varying reference trajectories are demonstrated using Lyapunov analysis. In addition, adaptation laws for the NNs weights of the proposed control systems are derived from the above procedure. The main contributions of this paper are summarized as follows:

1. Stabilization for fully actuated systems is well established in terms of the time-varying trajectories through adaptive control. However, its application and extension to the UMSs are not straightforward. This paper proposes the adaptive control schemes to encompass the conventional approaches and stabilize the UMSs' state space through design of auxil- iary control variables that contain $\mathrm{NN}$ approximator and robust compensator.

2. The parametric uncertainties and the matched and mismatched disturbances are considered in the design of the adaptive control schemes, which feature a generic model for the studies on underactuated systems. It is noted that the mismatched disturbances have been neglected in most of the existing approaches for the tracking control of UMSs.

3. Employing the adaptive control approach, combined with variable structure and NNs, exact values of the system base parameters are not required to be known a priori.

4. Designing robust compensators to counteract the matched and mismatched disturbances, and function approximation error of $\mathrm{NNs}$ and nonlinear frictions can reduce the tracking error as small as desired in finite time through selecting appropriate parameters for the controller.

The rest of this paper is organized as follows. Notations, assumptions, system dynamic model for UMSs and preliminaries are presented in Sect. 2. Section 3 gives the main theoretical results concerning the adaptive NN tracking control systems design for a UMSs. Validations of the effectiveness of the proposed approaches are presented in Sect. 4 through simulation studies on an underactuated manipulator and a vibro-driven mobile system. Finally, concluding remarks and perspectives are given in Sect. 5.

\section{Preliminaries and problem description}

\subsection{Notations}

Let $\|$.$\| denote any suitable vector Euclidean norm.$ Specifically, $\|\cdot\|_{p}$ represents the $p$-norm of a given vector. The Frobenius norm of the given matrix $H=$ $\left[h_{i j}\right] \in \mathcal{R}^{n \times m}$ is defined as $\|H\|_{F}^{2}=\operatorname{Tr}\left(H^{T} H\right)=$ $\operatorname{Tr}\left(H H^{T}\right)=\sum_{i, j} h_{i j}^{2}$ with $\operatorname{Tr}($.$) denoting the trace$ operator. The Frobenius norm is associated with the 2-norm in a manner that $\|H x\|_{2} \leq\|H\|_{F}\|x\|_{2}$ with $H \in \mathcal{R}^{n \times m}$ and $x \in \mathcal{R}^{m}$. The trace operator has the property of $\mathrm{A}^{T} B=\operatorname{Tr}\left(A B^{T}\right)$ with $\forall A, B \in \mathcal{R}^{n}$. $\lambda_{\min }$ (.) and $\lambda_{\max }$ (.) are, respectively, the minimum and maximum eigenvalue of the given matrix. $I_{n}$ represents the identity matrix of dimension $n \times n$. 
2.2 Dynamic model and properties

The dynamics of $n$-DOF UMSs can be expressed in the generalized coordinates via the Euler-Lagrangian's approach, given by

$$
\begin{aligned}
& D(q, \alpha) \ddot{q}+C(q, \dot{q}, \alpha) \dot{q}+G(q, \alpha)+F_{v}(\alpha) \dot{q} \\
& \quad+F_{c}(q, \dot{q}, \alpha)+\tau_{d}=B(q) \tau
\end{aligned}
$$

where $q=\left[q_{1}, \ldots, q_{n}\right]^{\mathrm{T}} \in \mathcal{R}^{n}$ describes the vector of generalized configurations, $\alpha \in \mathcal{R}^{p}$ is the vector of unknown parameters of the underactuated system, mainly including the initial parameters and possible loading parameters ( $p$ indicates the number of uncertain parameters), $D(q, \alpha) \in \mathcal{R}^{n \times n}$ is the inertial matrix, $C(q, \dot{q}, \alpha) \in \mathcal{R}^{n \times n}$ represents the centripetal and Coriolis matrix, $G(q, \alpha) \in \mathcal{R}^{n}$ denotes the gravitational torque/force, $F_{v}(\alpha) \in \mathcal{R}^{n \times n}$ is the viscous friction coefficients, $F_{c}(q, \dot{q}, \alpha) \in \mathcal{R}^{n}$ models the nonlinear friction torques, $\tau_{d}$ denotes the unknown disturbances and unmodelled dynamics which are bounded, $B(q) \in \mathcal{R}^{n \times(n-m)}$ represents the input transformation matrix and $\tau \in \mathcal{R}^{n-m}$ is the vector of control inputs to be constructed to obtain specific control objectives.

The Lagrangian dynamic model of the UMSs described by (1) has the following beneficial properties $[6,45,46]$ that are employed in the design and analysis of the control schemes in this paper:

Property 1 The inertia matrix $D(q, \alpha)$ is symmetric and positive definite, i.e. $D(q, \alpha)=D^{T}(q, \alpha)$; it is uniformly positive definite and has upper and lower boundaries, which implies

$$
\begin{aligned}
0 & <\lambda_{\min }(\alpha)\|x\|^{2} \leq x^{T} D(q, \alpha) x \leq \lambda_{\max }(\alpha)\|x\|^{2} \\
& <+\infty, \forall x \in \mathcal{R}^{n-m}
\end{aligned}
$$

Property 2 The centripetal and Coriolis term $C(q, \dot{q}, \alpha) \dot{q}$ is quadratic in the generalized velocity $\dot{q}$ and satisfies

$\|C(q, \dot{q}, \alpha) \dot{q}\| \leq \lambda_{3}(\alpha)\|\dot{q}\|^{2}$

where $\lambda_{3}(\alpha)$ is a bounded scalar constant.
Property 3 The above matrixes $D(q, \alpha)$ and $C(q, \dot{q}, \alpha)$ have the following skew-symmetric interconnection

$x^{T}[\dot{D}(q, \alpha)-2 C(q, \dot{q}, \alpha)] x=0, \forall x \in \mathcal{R}^{n-m}$

under an appropriate definition of $C(q, \dot{q}, \alpha)$. This property is a matrix version of energy conservation.

Property 4 The gravitational torquelforce $G(q, \alpha)$ is bounded and satisfies

$\|G(q, \alpha)\| \leq \lambda_{4}(\alpha)$

where $\lambda_{4}(\alpha)$ is a bounded constant.

Property 5 The dynamic model (1) can be rewritten in a linear form with respect to an appropriate selection of the system's initial parameters and load parameters $\alpha$. Furthermore, there exist a regressor matrix $Y(q, \dot{q}, \ddot{q})$ and a vector $Y_{0}(q, \dot{q}, \ddot{q})$ containing known functions, given as follows:

$$
\begin{aligned}
& D(q, \alpha) \ddot{q}+C(q, \dot{q}, \alpha) \dot{q}+G(q, \alpha)+F_{v}(\alpha) \dot{q} \\
& \quad+F_{c}(q, \dot{q}, \alpha)=Y(q, \dot{q}, \ddot{q}) \alpha+Y_{0}(q, \dot{q}, \ddot{q})
\end{aligned}
$$

where $Y(.) \in R^{(n-m) \times p}$ is the regressor matrix containing known functions.

Remark 1 Based on Property 5, we introduce $\hat{\alpha}$ be the time-varying estimation of $\alpha$, and define $\hat{D}, \hat{C}, \hat{G}, \hat{F}_{v}$ and $\hat{F}_{c}$ be the corresponding affine matrices, respectively, estimated from $D, C, G, F_{v}$ and $F_{c}$ through substitution $\hat{\alpha}$ for the real $\alpha$. Then, the linear parametrizability is given by

$$
\begin{aligned}
& \tilde{D}(q, \alpha) \ddot{q}+\tilde{C}(q, \dot{q}, \alpha) \varrho+\tilde{G}(q, \alpha) \\
& \quad+\tilde{F}_{v}(\alpha) \varrho+\tilde{F}_{c}(q, \dot{q}, \alpha) \\
& =Y(q, \dot{q}, \varrho, \ddot{q}) \tilde{\alpha}+Y_{0}(q, \dot{q}, \varrho, \ddot{q})
\end{aligned}
$$

where $\tilde{\alpha}(t)=\hat{\alpha}(t)-\alpha$ is the parameter estimation error, $\varrho \in \mathcal{R}^{n}$ is an arbitrary vector and $\tilde{D}, \tilde{C}, \tilde{G}, \tilde{F}_{v}, \tilde{F}_{c}$ represent the corresponding affine matrices of estimation errors in the presence of the parameter estimation error $\tilde{\alpha}$.

Remark 2 Concretely, the unmodelled friction torque/ force $F$ in (1) can be partitioned into two aspects as

$$
F=F_{v}(\alpha) \dot{q}+F_{c}(q, \dot{q}, \alpha)
$$




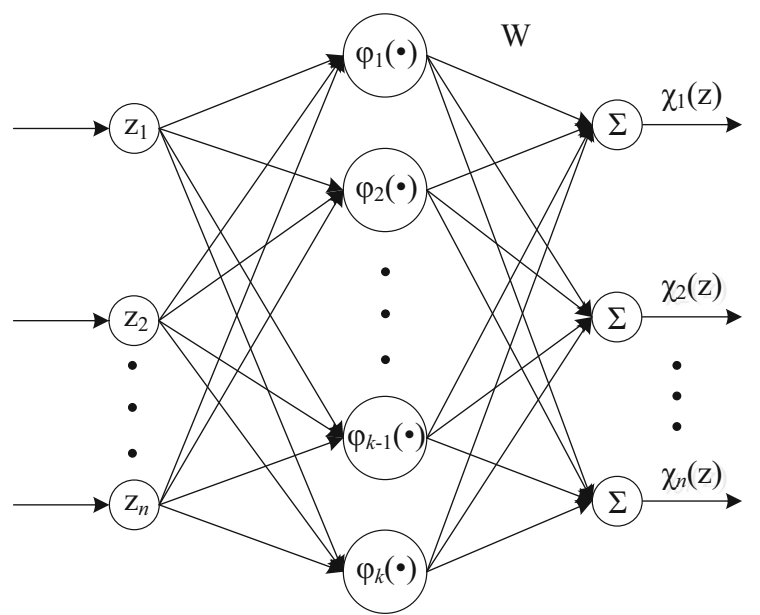

Input Layer Neuron Layer Output Layer

Fig. 1 Structure of the RBFNN

where $F_{v}(\alpha) \dot{q}=\left[F_{v 1}(\alpha) \dot{q}_{1}, F_{v 2}(\alpha) \dot{q}_{2}, \ldots, F_{v n}(\alpha)\right.$ $\left.\dot{q}_{n}\right]^{T}$ is the viscous friction torque describing the linear part and $F_{c}(q, \dot{q}, \alpha)=\left[F_{c 1}\left(q_{1}, \dot{q}_{1}, \alpha\right), F_{c 2}\right.$ $\left.\left(q_{2}, \dot{q}_{2}, \alpha\right), \ldots, F_{c n}\left(q_{n}, \dot{q}_{n}, \alpha\right)\right]^{T}$ denotes the nonlinear friction torques.

Definition 1 [47] UMSs' DOF contains two subsets, including the collocated subset whose cardinality equals the number of control inputs and encompasses the actuated DOF, and the non-collocated subset contains the rest of the DOF which are passive.

Assumption 1 It is assumed in this paper that the matched and mismatched external disturbances are bounded.

Assumption 2 It is assumed that each subsystem is equipped with encoder and tachometer for the position and velocity measurement.

\subsection{RBFNN approximation}

The structure of RBFNN is presented in Fig. 1. The universal approximation capability of RBFNN towards any continuous nonlinear function $\chi(z): \mathcal{R}^{n} \rightarrow \mathcal{R}$ over a compact set $\Omega_{z}$ has been well established, which can be expressed as

$$
\begin{aligned}
\chi(z)= & W^{* T} \phi(z)+\varepsilon(z) \forall z \in \Omega_{z} \subset \mathcal{R}^{n}, \\
& \|\varepsilon(z)\| \leq \varepsilon_{N}
\end{aligned}
$$

where $z \in \Omega_{z} \subset \mathcal{R}^{n}$ denotes the input vector of dimension $n, \chi(z)$ is the unknown function to be approximated, $W^{*}=\left[W_{1}^{*}, W_{2}^{*}, \ldots, W_{k}^{*}\right]^{T} \in \mathcal{R}^{k}$ is the bounded ideal synaptic weight vector with dimension (or the NN node number) $k>1$ (i.e. $\forall$ positive constant $W_{N}$ such that $\left\|W_{k}^{*}\right\| \leq W_{N}$ and $\operatorname{tr}\left\{W_{k}^{*^{T}} W_{k}^{*}\right\} \leq$ $\left.W_{N}\right), \varepsilon(z) \in \mathcal{R}$ is a bounded approximation error over the compact set, $\varepsilon_{N}$ is an upper bound (positive constant) of the approximation error which satisfies $\varepsilon_{N}=\sup \left\|\hat{\chi}\left(z, W^{*}\right)-\chi(z)\right\|$ and $\phi(z)=$ $\left[\phi_{1}(z), \phi_{2}(z), \ldots, \phi_{k}(z)\right]^{T}$ is the NN basis function which is conventionally chosen as Gaussian functions as

$$
\phi_{i}(z)=\exp \left(-\frac{\left\|z-C_{i}\right\|^{2}}{2 b_{i}^{2}}\right), i=1,2, \ldots, k
$$

where vector $C_{i}$ and $b_{i}$ represent the centre and the width of the $i$-th receptive field.

The Gaussian function is chosen as NN basis function; it is well known that given a sufficient number of NNs nodes and properly adopted centres and the widths of the node, RBFNN is able to approximate any unknown nonlinearities to arbitrarily close to a compact set with any desired accuracy. Note that the approximation error $\varepsilon(z)$ decreases along with the increase in the number of $\mathrm{NN}$ node $k$.

It is noted that the bounded ideal weight matrix $W^{*}$ is merely a quantity utilized for analysis purposes, whilst in practical control applications, the estimate $\hat{W}$ of $W^{*}$ is utilized for practical approximation of unknown nonlinear function $\chi(z)$. As such, the estimation of $\chi(z)$ is represented by

$\hat{\chi}(z)=\hat{W}^{T} \phi(z)$

Based on the NN defined by (11), approximation error of the nonlinear function can be described as

$\chi(z)-\hat{\chi}(z)=\tilde{W}^{T} \phi(z)+\varepsilon(z)$

where $\tilde{W}=W^{*}-\hat{W}$.

Assumption $3 \hat{\chi}\left(z, W^{*}\right)$ is the output of the NNs and continuous; there exists a sufficient small positive constant such that

$\max \left\|\hat{\chi}\left(z, W^{*}\right)-\chi(z)\right\| \leq \varepsilon_{0}$ 
where $W^{*}$ is typically defined as the optimal value of $W$ such that the approximation error $\varepsilon(z)$ could be minimized for all $z \in \Omega_{z}$ as

$$
W^{*}:=\arg \min _{W \in \mathcal{R}^{k}}\left\{\sup _{z \in \Omega_{z}}\left\|\chi(z)-W^{* T} \phi(z)\right\|\right\}
$$

\section{Control system design and stability analysis}

It is assumed that for system (1), there are only $m$ control inputs that are equipped with actuators; then, the generalized coordinate vector $q$ can be partitioned into collocated and non-collocated vectors as

$q:=\left[q_{c} q_{n}\right]^{T}$

where $q_{c} \in \mathcal{R}^{m}$ and $q_{n} \in \mathcal{R}^{n-m}$ denote the actuated and unactuated coordinate vector, respectively. The subscripts " $c$ " and " $n$," respectively, indicate collocated and non-collocated subsets.

Without loss of generality, the underactuated system (1) can be rewritten into a partitioned form as

$$
\left\{\begin{array}{l}
D_{c c}(q, \alpha) \ddot{q}_{c}+D_{c n}(q, \alpha) \ddot{q}_{n}+C_{c c}(q, \dot{q}, \alpha) \dot{q}_{c} \\
\quad+C_{c n}(q, \dot{q}, \alpha) \dot{q}_{n}+G_{c}(q, \alpha)+F_{v c}(\alpha) \dot{q}_{c} \\
\quad+F_{c c}(q, \dot{q}, \alpha)+\tau_{\mathrm{dc}}=\tau \\
D_{n c}(q, \alpha) \ddot{q}_{c}+D_{n n}(q, \alpha) \ddot{q}_{n}+C_{n c}(q, \dot{q}, \alpha) \dot{q}_{c} \\
\quad+C_{n n}(q, \dot{q}, \alpha) \dot{q}_{n}+G_{n}(q, \alpha) \\
\quad+F_{v n}(\alpha) \dot{q}_{n}+F_{c n}(q, \dot{q}, \alpha)+\tau_{d n}=0
\end{array}\right.
$$

where $\tau_{\mathrm{dc}}$ and $\tau_{d n}$ denote the bounded unknown disturbances and unmodelled dynamics to the collocated and non-collocated subsets, respectively.

Let the reference trajectories for the collocated and non-collocated subsets be descried by the vector-valued functions $\left\|q_{c d}\right\|_{\infty} \leq \vartheta_{1}$ and $\left\|q_{n d}\right\|_{\infty} \leq \vartheta_{2}$, respectively, and assume that these functions are bounded in norm and uniformly continuous on $\mathcal{R}^{+}$, and homogenously on the same set, its first- and second-order derivatives are bounded, well defined and uniformly continuous. Introduce the trajectory tracking error as

$\tilde{q}_{c}=q_{c}-q_{c d}, \tilde{q}_{n}=q_{n}-q_{n d}$

which is to be stabilized to zero without the knowledge of the system parameters $\alpha . \vartheta_{1}$ and $\vartheta_{2}$ are positive upper bounds of the desired reference trajectories. Noting that the design of $\vartheta_{1}$ and $\vartheta_{2}$ has to satisfy the zero dynamics based on the non-holonomic dynamics, we have

$$
\begin{aligned}
& D_{n c}(q, \alpha) \ddot{\vartheta}_{1}+D_{n n}(q, \alpha) \ddot{\vartheta}_{2}+C_{n c}(q, \dot{q}, \alpha) \dot{\vartheta}_{1} \\
& \quad+C_{n n}(q, \dot{q}, \alpha) \dot{\vartheta}_{2}+G_{n}(q, \alpha) \\
& \quad+F_{v n}(\alpha) \dot{\vartheta}_{2}+F_{c n}(q, \dot{q}, \alpha)+\tau_{d n}=0
\end{aligned}
$$

In the following, auxiliary kinematic vector variables $\varrho=\left[\varrho_{c} \varrho_{n}\right]^{T}$ and $\delta=\left[\delta_{c} \delta_{n}\right]^{T}$ are defined as

$\varrho_{c}=\dot{q}_{c d}-\Lambda_{c} \tilde{q}_{c}$,

$\varrho_{n}=\dot{q}_{n d}-\Lambda_{n} \tilde{q}_{n}$

$\delta_{c}=\dot{q}_{c}-\varrho_{c}=\dot{\tilde{q}}_{c}+\Lambda_{c} \tilde{q}_{c}$,

$\delta_{n}=\dot{q}_{n}-\varrho_{n}=\dot{\tilde{q}}_{n}+\Lambda_{n} \tilde{q}_{n}$

where $\varrho_{c}, \delta_{c} \in \mathcal{R}^{m}$ and $\varrho_{n}, \delta_{n} \in \mathcal{R}^{n-m} . \delta$ denotes the filtered error signal and describes the measure of tracking accuracy, $\varrho$ is referred to as vector of the reference trajectory, $\Lambda=\operatorname{diag}\left[\Lambda_{c} I_{m \times m}, \Lambda_{n} I_{(n-m) \times(n-m)}\right]$ with $\Lambda_{c}$ and $\Lambda_{n}$ be positive constants selected by designers. $I_{i \times i}$ denotes $i \times i$ identity matrix. It is noted that the error dynamics of the underactuated systems can be obtained by firstly introducing the tracking error from collocated and non-collocated loops and then filtering out the error signals. In this regard, we can encompass the conventional adaptive control approaches and stabilize the state space of underactuated systems. The choice of $\Lambda_{c}>0$ and $\Lambda_{n}>0$ guarantees that (20) is an exponentially stable system for $q$. Therefore, the trajectory $q$ converges to an adjacent of $q_{d}$ exponentially fast as long as the control system drives $\delta$ to an adjacent of zero.

Applying the defined variables in the system dynamics (17), we have

$$
\left\{\begin{array}{l}
D_{c c}(q, \alpha)\left(\dot{\delta}_{c}+\dot{\varrho}_{c}\right)+D_{c n}(q, \alpha)\left(\dot{\delta}_{n}+\dot{\varrho}_{n}\right) \\
\quad+C_{c c}(q, \dot{q}, \alpha)\left(\delta_{c}+\varrho_{c}\right)+C_{c n}(q, \dot{q}, \alpha)\left(\delta_{n}\right. \\
\left.\quad+\varrho_{n}\right)+G_{c}(q, \alpha)+F_{v c}(\alpha) \dot{q}_{c}+F_{c c}(q, \dot{q}, \alpha) \\
\quad+\tau_{\mathrm{dc}}=\tau \\
D_{n c}(q, \alpha)\left(\dot{\delta}_{c}+\dot{\varrho}_{c}\right)+D_{n n}(q, \alpha)\left(\dot{\delta}_{n}+\dot{\varrho}_{n}\right) \\
\quad+C_{n c}(q, \dot{q}, \alpha)\left(\delta_{c}+\varrho_{c}\right)+C_{n n}(q, \dot{q}, \alpha)\left(\delta_{n}+\varrho_{n}\right) \\
\quad+G_{n}(q, \alpha)+F_{v n}(\alpha) \dot{q}_{n}+F_{c n}(q, \dot{q}, \alpha)+\tau_{d n}=0
\end{array}\right.
$$


The corresponding lumped error equation can be yielded as

$$
\left\{\begin{array}{l}
D_{c c}(q, \alpha) \dot{\delta}_{c}+D_{c n}(q, \alpha) \dot{\delta}_{n}+C_{c c}(q, \dot{q}, \alpha) \delta_{c} \\
\quad+C_{c n}(q, \dot{q}, \alpha) \delta_{n}+\tau_{\mathrm{dc}}=\tau \\
\quad-Y_{c}\left(q, \dot{q}, \dot{\varrho}_{c}, \dot{\varrho}_{n}, \varrho_{c}, \varrho_{n}\right) \alpha_{c} \\
D_{n c}(q, \alpha) \dot{\delta}_{c}+D_{n n}(q, \alpha) \dot{\delta}_{n}+C_{n c}(q, \dot{q}, \alpha) \delta_{c} \\
\quad+C_{n n}(q, \dot{q}, \alpha) \delta_{n}+\tau_{d n}=-\chi(\mathrm{z})
\end{array}\right.
$$

where $Y_{c}\left(q, \dot{q}, \dot{\varrho}_{c}, \dot{\varrho}_{n}, \varrho_{c}, \varrho_{n}\right) \alpha_{c}=D_{c c}(q, \alpha) \dot{\varrho}_{c}+$ $D_{c n}(q, \alpha) \dot{\varrho}_{n}+C_{c c}(q, \dot{q}, \alpha) \varrho_{c}+C_{c n}(q, \dot{q}, \alpha) \varrho_{n}+$ $G_{c}(q, \alpha)+F_{v c}(\alpha) \dot{q}_{c}+F_{c c}(q, \dot{q}, \alpha), \quad \chi(\mathrm{z})=$ $D_{n c}(q, \alpha) \dot{\varrho}_{c}+D_{n n}(q, \alpha) \dot{\varrho}_{n}+C_{n c}(q, \dot{q}, \alpha) \varrho_{c}+$ $C_{n n}(q, \dot{q}, \alpha) \varrho_{n}+G_{n}(q, \alpha)+F_{v n}(\alpha) \dot{q}_{n}+F_{c n}(q, \dot{q}, \alpha)$, and $\alpha_{c}=\hat{\alpha}_{c}-\tilde{\alpha}_{c}, \alpha_{n}=\hat{\alpha}_{n}-\tilde{\alpha}_{n}$. The input $\chi(\mathrm{z})$ is adopted as $\mathrm{z}=\left[\tilde{q}^{T}, \dot{\tilde{q}}^{T}, q_{d}^{T}, \dot{q}_{d}^{T}, \ddot{q}_{d}^{T}\right]$.

The estimation of nonlinear function $\chi(\mathrm{z})=$ $-Y_{n}\left(q, \dot{q}, \dot{\varrho}_{c}, \dot{\varrho}_{n}, \varrho_{c}, \varrho_{n}\right) \alpha_{n}$ is expressed as

$$
\hat{\chi}(z)=\hat{W}^{T} \phi(z)
$$

where $\hat{W}$ is the $\mathrm{NN}$ adaptation law, $\phi(z)$ is the basis function.

Accordingly, (22) evolves to the following form

$$
\left\{\begin{array}{l}
D_{c c}(q, \alpha) \dot{\delta}_{c}+D_{c n}(q, \alpha) \dot{\delta}_{n}+C_{c c}(q, \dot{q}, \alpha) \delta_{c} \\
+C_{c n}(q, \dot{q}, \alpha) \delta_{n}+\tau_{\mathrm{dc}}=\tau \\
\quad-Y_{c}\left(q, \dot{q}, \dot{\varrho}_{c}, \dot{\varrho}_{n}, \varrho_{c}, \varrho_{n}\right) \alpha_{c} \\
D_{n c}(q, \alpha) \dot{\delta}_{c}+D_{n n}(q, \alpha) \dot{\delta}_{n}+C_{n c}(q, \dot{q}, \alpha) \delta_{c} \\
+C_{n n}(q, \dot{q}, \alpha) \delta_{n}+\tau_{d n}=\hat{W}^{T} \phi+\tilde{W}^{T} \phi+\varepsilon
\end{array}\right.
$$

where $\tilde{W}=W^{*}-\hat{W}$.

Concretely, with these derivations, the adaptive control problem for underactuated systems can be formulated as: given the reference trajectories $q_{d} \in \mathcal{R}^{n}$, finding a nonlinear control law for $\tau$ such that for any $q(0) \in \mathcal{R}^{n}$ subjecting to parameter uncertainty and external matched and mismatched disturbances, the tracking error $\tilde{q}$ and its derivative converge to zero in finite time as $t \rightarrow \infty$.

The following theorem presents NNs-based control schemes that ensure the convergence of the closed-loop signals.

Theorem 1 Consider the dynamic properties, assumptions and definitions, and apply the following control laws to the uncertain underactuated system (24) $\tau=\tau_{c}+\tau_{n}$

$\tau_{c}=Y_{c} \hat{\alpha}_{c}-K_{1} \delta_{c}-\xi$,

$\tau_{n}=-\operatorname{sgn}\left(\delta_{c}\right)\left\|\delta_{n}\right\||\eta|-K_{2} \operatorname{sgn}\left(\delta_{c}\right)\left\|\delta_{n}\right\|$

where the Adaptation Algorithm 1 for the collocated subsystem is designed as

$\dot{\hat{\alpha}}_{c}=-\Gamma Y_{c} \delta_{c}$

and the auxiliary input $\eta$ in $(25 b)$ is constructed as

$\dot{\eta}=\eta^{\frac{1}{2 n+1}}\left(-K_{3}\left\|\delta_{n}\right\|^{2}-\left\|\delta_{n}\right\| \hat{W}^{T} \phi+\delta_{n}^{T} \zeta\right)$

with robust compensator $\zeta$ for the non-collocated subsystem designed as

$\zeta=-\frac{\delta_{n}}{\left\|\delta_{n}\right\|+\mu} \kappa$

and its adaptation law

$\dot{\kappa}=\frac{\left\|\delta_{n}\right\|^{2}}{\left\|\delta_{n}\right\|+\mu}$

where $K_{1} \in \mathcal{R}^{m \times m}, K_{2}, K_{3} \in \mathcal{R}^{(n-m) \times(n-m)}$ are diagonal, constant positive definite matrixes and $\Gamma \in$ $\mathcal{R}^{p \times p}$ are positive definite matrixes. $\xi$ and $\zeta$ are auxiliary robust compensators designed later for convenience of stability analysis of the closed-loop system, and they are designed to compensate for matched and mismatched disturbances, and function approximation error of NNs and nonlinear frictions. $\mu>0$ is selected in a manner that $\int_{0}^{\infty} \mu \mathrm{d} t<\infty$. Then, the following conclusions hold:

(1) $\operatorname{tr}\left\{\hat{W}^{T} \hat{W}\right\} \leq W_{N}$ holds.

(2) The control objective of global asymptotically stabilization can be achieved;

(3) All signals within the closed-loop system are bounded, and the trajectory tracking errors $\tilde{q}$ and $\dot{\tilde{q}}$ will converge to zero asymptotically.

Proof Consider a candidate Lyapunov function as follows

$$
\begin{aligned}
V= & \frac{1}{2} \delta^{T} D \delta+\frac{1}{2} \tilde{\alpha}_{c}^{T} \Gamma^{-1} \tilde{\alpha}_{c}+\frac{1}{2} \operatorname{tr}\left\{\tilde{W}^{T} \Upsilon^{-1} \tilde{W}\right\} \\
& +\frac{2 n+1}{2 n} \eta^{\frac{2 n}{2 n+1}}+\frac{1}{2}\left(\kappa-\varepsilon_{T}\right)^{2}
\end{aligned}
$$


where $\varepsilon_{T} \geq\left\|\varepsilon-\tau_{d n}\right\|$ denotes the upper bound of the mismatched disturbance and approximation error.

Differentiating both sides of (26) and applying the control laws (25) yield

$$
\begin{aligned}
& \dot{V}=\delta^{T}\left(\left[\begin{array}{c}
\tau-Y_{c} \alpha_{c} \\
W^{T} \phi+\varepsilon
\end{array}\right]-\tau_{d}\right)+\dot{\hat{\alpha}}_{c}^{T} \Gamma^{-1} \tilde{\alpha}_{c} \\
& +\operatorname{tr}\left\{\tilde{W}^{T} \Upsilon^{-1} \dot{\tilde{W}}\right\}+\left(\kappa-\varepsilon_{T}\right) \dot{\kappa}+\eta^{\frac{-1}{2 n+1}} \dot{\eta} \\
& =\left[\delta_{c}^{T} \delta_{n}^{T}\right] \\
& {\left[\begin{array}{c}
-Y_{c} \tilde{\alpha}_{c}-K_{1} \delta_{c}-\operatorname{sgn}\left(\delta_{c}\right)\left\|\delta_{n}\right\||\eta|-\xi-K_{2} \operatorname{sgn}\left(\delta_{c}\right)\left\|\delta_{n}\right\| \\
W^{T} \phi+\varepsilon
\end{array}\right]} \\
& -\delta^{T} \tau_{d}+\dot{\hat{\alpha}}_{c}^{T} \Gamma^{-1} \tilde{\alpha}_{c}+\operatorname{tr}\left\{\tilde{W}^{T} \Upsilon^{-1} \dot{\tilde{W}}\right\} \\
& +\left(\kappa-\varepsilon_{T}\right) \dot{\kappa}+\eta^{\frac{-1}{2 n+1}} \dot{\eta} \\
& =-\delta_{c}^{T} K_{1} \delta_{c}-\delta_{c}^{T} K_{2} \operatorname{sgn}\left(\delta_{c}\right)\left\|\delta_{n}\right\|-\delta_{c}^{T} \xi \\
& -\delta_{c}^{T} \operatorname{sgn}\left(\delta_{c}\right)\left\|\delta_{n}\right\||\eta|-\delta^{T} \tau_{d}+\delta_{n}^{T}\left(W^{T} \phi+\varepsilon\right) \\
& +\operatorname{tr}\left\{\tilde{W}^{T} \Upsilon^{-1} \dot{\tilde{W}}\right\}+\left(\kappa-\varepsilon_{T}\right) \dot{\kappa}+\eta^{\frac{-1}{2 n+1}} \dot{\eta} \\
& =-\delta_{c}^{T} K_{1} \delta_{c}-K_{2}\left\|\delta_{c}\right\|\left\|\delta_{n}\right\|-\left\|\delta_{c}\right\|\left\|\delta_{n}\right\||\eta| \\
& +\delta_{n}^{T} \varepsilon+\delta_{n}^{T} W^{T} \phi-\delta_{c}^{T} \xi-\delta^{T} \tau_{d}+\operatorname{tr}\left\{\tilde{W}^{T} \Upsilon^{-1} \dot{\tilde{W}}\right\} \\
& +\left(\kappa-\varepsilon_{T}\right) \dot{\kappa}+\eta^{\frac{-1}{2 n+1}} \dot{\eta} \\
& =-\delta_{c}^{T} K_{1} \delta_{c}-K_{2}\left\|\delta_{c}\right\|\left\|\delta_{n}\right\|-\left\|\delta_{c}\right\|\left\|\delta_{n}\right\||\eta| \\
& -\delta_{c}^{T} \xi-\delta^{T} \tau_{d}+\delta_{n}^{T} \varepsilon+\delta_{n}^{T} W^{T} \phi+\operatorname{tr}\left\{\tilde{W}^{T} \Upsilon^{-1} \dot{\tilde{W}}\right\} \\
& -K_{3}\left\|\delta_{n}\right\|^{2}+\left(\kappa-\varepsilon_{T}\right) \dot{\kappa}-\left\|\delta_{n}\right\| \hat{W}^{T} \phi-\delta_{n}^{T} \zeta \\
& =-\delta_{c}^{T} K_{1} \delta_{c}-K_{2}\left\|\delta_{c}\right\|\left\|\delta_{n}\right\|-\left\|\delta_{c}\right\|\left\|\delta_{n}\right\||\eta| \\
& +\delta_{n}^{T}\left(\varepsilon-\tau_{d n}\right)-\delta_{c}^{T} \xi-\delta_{c}^{T} \tau_{\mathrm{dc}}-K_{3}\left\|\delta_{n}\right\|^{2} \\
& -\frac{\left\|\delta_{n}\right\|^{2}}{\left\|\delta_{n}\right\|+\mu} \kappa+\left(\kappa-\varepsilon_{T}\right) \dot{\kappa} \\
& +\operatorname{tr}\left\{\tilde{W}^{T} \Upsilon^{-1}\left(\dot{\tilde{W}}+\Upsilon \delta_{n}^{T} \phi\right)\right\} \\
& \leq-\delta_{c}^{T} K_{1} \delta_{c}-K_{2}\left\|\delta_{c}\right\|\left\|\delta_{n}\right\|-\left\|\delta_{c}\right\|\left\|\delta_{n}\right\||\eta| \\
& +\left\|\delta_{n}\right\| \varepsilon_{T}-\delta_{c}^{T} \xi-\delta_{c}^{T} \tau_{\mathrm{dc}}-K_{3}\left\|\delta_{n}\right\|^{2}-\frac{\left\|\delta_{n}\right\|^{2}}{\left\|\delta_{n}\right\|+\mu} \kappa \\
& +\left(\kappa-\varepsilon_{T}\right) \dot{\kappa}+\operatorname{tr}\left\{\tilde{W}^{T} \Upsilon^{-1}\left(\dot{\tilde{W}}+\Upsilon \delta_{n}^{T} \phi\right)\right\}
\end{aligned}
$$

Towards the parameter drifting problem, the neural weight adaptation law for $\hat{W}$ is constructed based on the projection algorithm, given by

$$
\dot{\hat{W}}=-\dot{\tilde{W}}=\left\{\begin{array}{l}
\Upsilon \phi \delta_{n}^{T}-\frac{\delta_{n}^{T} \hat{W}^{T} \Upsilon \phi \hat{W}}{W_{N}}, \quad \text { if } \operatorname{tr}\left\{\hat{W}^{T} \hat{W}\right\} \\
=W_{N} \text { and } \delta_{n}^{T} \hat{W}^{T} \phi \leq 0 ; \\
\Upsilon \phi \delta_{n}^{T}, \text { if } \operatorname{tr}\left\{\hat{W}^{T} \hat{W}\right\}<W_{N} \text { or } \\
\text { if } \operatorname{tr}\left\{\hat{W}^{T} \hat{W}\right\}=W_{N} \text { and } \\
\delta_{n}^{T} \hat{W}^{T} \phi>0 .
\end{array}\right.
$$

Corollary 1 Let $V_{t r 1} \triangleq \operatorname{tr}\left\{\hat{W}^{T} \hat{W}\right\}$ and $V_{t r 2} \triangleq$ $\operatorname{tr}\left\{\tilde{W}^{T} \Upsilon^{-1}\left(\dot{\tilde{W}}+\Upsilon \delta_{n}^{T} \phi\right)\right\}$ and apply weight adaptation law (28), then the following results hold for the boundedness of $\hat{W}$

(1) $V_{t r 1} \leq W_{N}$

(2) $V_{t r 2} \leq 0$

Proof (1) Recalling (28), it is evident that

(a) If $V_{t r 1}=W_{N}$ and $\delta_{n}^{T} \hat{W}^{T} \phi>0$,

$$
\begin{aligned}
& \dot{V}_{t r 1}=2 \operatorname{tr}\left\{\hat{W}^{T} \dot{\hat{W}}\right\}=2 \operatorname{tr}\left\{\hat{W}^{T} \Upsilon \phi \delta_{n}^{T}\right\}- \\
& 2 \delta_{n}^{T} \hat{W}^{T} \Upsilon \phi=0 .
\end{aligned}
$$

(b) If $V_{t r 1}=W_{N}$ and $\delta_{n}^{T} \hat{W}^{T} \phi \leq 0, \dot{V}_{t r 1}=$ $2 \operatorname{tr}\left\{\hat{W}^{T} \Upsilon \phi \delta_{n}^{T}\right\}<0$.

(c) If $V_{t r 1}<W_{N}$, the result 1) holds by itself. (2) Adopting $\dot{\hat{W}}$ in (28), it is apparent that

(a) If $V_{t r 1}=W_{N}$ and $\delta_{n}^{T} \hat{W}^{T} \Upsilon \phi>0$,

$$
\begin{aligned}
V_{t r 2}= & \frac{\delta_{n}^{T} \hat{W}^{T} \Upsilon \phi}{W_{N}} \operatorname{tr}\left\{\hat{W}^{T} \hat{W}\right\} \\
& \leq \frac{\delta_{n}^{T} \hat{W}^{T} \Upsilon \phi}{W_{N}}\left(\frac{1}{2} \operatorname{tr}\left\{W^{*^{T}} W^{*}\right\}-\frac{1}{2} W_{N}\right) \\
& \leq 0
\end{aligned}
$$

(b) If $\dot{\hat{W}}=\Upsilon \phi \delta_{n}^{T}$, we have $V_{t r 2}=0$. This completes the proof of Corollary 1 .

Substituting (30) into (27), the time derivative of Lyapunov candidate function becomes

$$
\begin{aligned}
\dot{V} \leq & -\delta_{c}^{T} K_{1} \delta_{c}-\left\|\delta_{c}\right\|\left\|\delta_{n}\right\||\eta|-K_{2}\left\|\delta_{c}\right\|\left\|\delta_{n}\right\|-\delta_{c}^{T} \xi \\
& -\delta_{c}^{T} \tau_{\mathrm{dc}}+\left\|\delta_{n}\right\| \varepsilon_{T} \\
& -\frac{\left\|\delta_{n}\right\|^{2}}{\left\|\delta_{n}\right\|+\mu} \kappa-K_{3}\left\|\delta_{n}\right\|^{2}+\left(\kappa-\varepsilon_{T}\right) \dot{\kappa} \\
= & -\delta_{c}^{T} K_{1} \delta_{c}-\left\|\delta_{c}\right\|\left\|\delta_{n}\right\||\eta|-K_{2}\left\|\delta_{c}\right\|\left\|\delta_{n}\right\| \\
& -\delta_{c}^{T} \xi-K_{3}\left\|\delta_{n}\right\|^{2}-\delta_{c}^{T} \tau_{\mathrm{dc}}+\left\|\delta_{n}\right\| \varepsilon_{T}
\end{aligned}
$$




$$
\begin{aligned}
& +\left(\kappa-\varepsilon_{T}\right)\left(\dot{\kappa}-\frac{\left\|\delta_{n}\right\|^{2}}{\left\|\delta_{n}\right\|+\mu}\right)-\frac{\left\|\delta_{n}\right\|^{2}}{\left\|\delta_{n}\right\|+\mu} \varepsilon_{T} \\
\leq & -\delta_{c}^{T} K_{1} \delta_{c}-\left\|\delta_{c}\right\|\left\|\delta_{n}\right\||\eta|-K_{2}\left\|\delta_{c}\right\|\left\|\delta_{n}\right\| \\
& -\delta_{c}^{T} \xi-K_{3}\left\|\delta_{n}\right\|^{2}-\delta_{c}^{T} \tau_{\mathrm{dc}} \\
& +\left\|\delta_{n}\right\| \varepsilon_{T}-\frac{\left\|\delta_{n}\right\|^{2}}{\left\|\delta_{n}\right\|+\mu} \varepsilon_{T} \\
= & -\delta_{c}^{T} K_{1} \delta_{c}-\left\|\delta_{c}\right\|\left\|\delta_{n}\right\||\eta|-K_{2}\left\|\delta_{c}\right\|\left\|\delta_{n}\right\| \\
& -\delta_{c}^{T} \xi-K_{3}\left\|\delta_{n}\right\|^{2}-\delta_{c}^{T} \tau_{\mathrm{dc}}+\frac{\left\|\delta_{n}\right\| \mu \varepsilon_{T}}{\left\|\delta_{n}\right\|+\mu} \\
\leq & -\delta_{c}^{T} K_{1} \delta_{c}-\left\|\delta_{c}\right\|\left\|\delta_{n}\right\||\eta|-K_{2}\left\|\delta_{c}\right\|\left\|\delta_{n}\right\| \\
& -\delta_{c}^{T} \xi-K_{3}\left\|\delta_{n}\right\|^{2}-\delta_{c}^{T} \tau_{\mathrm{dc}}+\mu \varepsilon_{T} \\
\leq & K_{1}\left\|\delta_{c}\right\|^{2}-K_{3}\left\|\delta_{n}\right\|^{2}-\delta_{c}^{T} \xi-\delta_{c}^{T} \tau_{\mathrm{dc}}+\mu \varepsilon_{T} \\
= & -\|\delta\|^{T} K\|\delta\|-\delta_{c}^{T} \xi-\delta_{c}^{T} \tau_{\mathrm{dc}}+\mu \varepsilon_{T}
\end{aligned}
$$

where $K=\left[\begin{array}{cc}K_{1} & 0 \\ 0 & K_{3}\end{array}\right]$.

When no disturbance exerts on the collocated sub$\operatorname{system}\left(\tau_{\mathrm{dc}}=0\right)$, i.e. the system is only subject to mismatched disturbances, we design the collocated robust compensator as $\xi=0$ and integrate both sides of (31) from $t=0$ to $t=T$ as

$V(T)-V(0) \leq-\int_{0}^{T}\|\delta\|^{T} K\|\delta\| \mathrm{d} t+\varepsilon_{T} \int_{0}^{T} \mu \mathrm{d} t$

Considering that $V(T) \geq 0$ and $\int_{0}^{\infty} \mu \mathrm{d} t<\infty$, we have

$$
\begin{gathered}
\lim _{T \rightarrow \infty} \sup \frac{1}{T} \int_{0}^{T}\|\delta\|^{2} \mathrm{~d} t \leq \frac{1}{K}\left(V(0)+\varepsilon_{T} \int_{0}^{T} \mu \mathrm{d} t\right) \\
\lim _{T \rightarrow \infty} \frac{1}{T}
\end{gathered}
$$

From the definition of the Lyapunov function $V$ in (26) and $\dot{V}$ derived from (31-33), the global uniform boundedness of the filtered tracking error $\delta_{c}$ for collocated subsystem and $\delta_{n}$ for non-collocated subsystem, the parameter estimation error $\tilde{W}$ is guaranteed. From the definition and assumption 1 of filtered tracking error $\delta$, it is evident that $\delta$ is bounded. The boundedness of control input is obvious from (25). It can be concluded that since $\delta=\left[\delta_{c} \delta_{n}\right]^{T} \in L_{2}^{n} \cap L_{\infty}^{n}, \delta_{c}$ and $\delta_{n}$ are continuous and $\delta_{c} \rightarrow 0, \delta_{n} \rightarrow 0$ as $t \rightarrow \infty$, and $\eta \in L_{\infty}$. From (25c), it can be shown that $\tilde{\alpha}_{c} \in L_{\infty}^{p}$. This in turn implies, based on property 1 and (25c), that $\dot{\delta} \in L_{\infty}^{n}, \ddot{q}=\left[\ddot{q}_{c} \ddot{q}_{n}\right]^{T} \in L_{\infty}^{n}$ and $\tilde{q}=\left[\tilde{q}_{c} \tilde{q}_{n}\right]^{T} \in L_{\infty}^{2 n}$. Therefore, $\tilde{q}_{c}$ and $\tilde{q}_{n}$ are uniformly continuous and $\tilde{q}=\left[\tilde{q}_{c} \tilde{q}_{n}\right]^{T} \in L_{\infty}^{2 n}$, and it is evident that $\tilde{q} \rightarrow 0$ as $t \rightarrow \infty$.

Remark 3 The NNs are adopted to approximate the mismatched system uncertainties, and the adaptive control algorithm is constructed to estimate the NN approximation error and the bounded mismatched disturbance. The combination of variable structure control, $\mathrm{NN}$ approximation and adaptive approach makes the constructed new controller more robust, and such errors resulting from trajectory tracking, parameter uncertainties, mismatched external disturbances and NN approximation are compensated.

For the case $\tau_{\mathrm{dc}} \neq 0$ and $\left\|\tau_{\mathrm{dc}}\right\|<\beta_{m}$, i.e. the system is subject to both matched and mismatched disturbances, one can only conclude that $\delta$ is bounded from (26) and (31), but $\tilde{\alpha}_{c}$ and $\tilde{W}$ may become unbounded as (31) merely contains a negative definite component of $\|\delta\|^{2}$ and no negative terms of $\tilde{\alpha}_{c}$ and $\tilde{W}$ are apparently included. As a result, the system may tend to be unstable. To improve the robustness of Theorem 1, the following adaptation algorithm is therefore proposed.

Adaptation Algorithm 2. Consider the following adaptation law

$\dot{\hat{\alpha}}_{c}=-\Gamma^{\prime} \tilde{\alpha}_{c}-\Gamma Y_{c} \delta_{c}$

Corollary 2 Consider the error equation (22) with the sliding surface designed in (20) under the adaptive NNs-based robust control law in (25), the following corollary holds: If adaptation algorithm 2 is adopted, the system error signals $\tilde{q}, \dot{\tilde{q}}$ and $\tilde{\alpha}$ converge to zero asymptotically. If $\tau_{\mathrm{dc}} \neq 0$ and $\left\|\tau_{\mathrm{dc}}\right\|<\beta_{m}$, then the system becomes globally uniformly ultimately stable and the boundedness depends on $\tau_{\mathrm{dc}}$.

Proof Adopting Adaptation Algorithm 2 in function (27), we have

$$
\begin{aligned}
\dot{V} & =\left[\delta_{c}^{T} \delta_{n}^{T}\right] \\
& {\left[\begin{array}{c}
-K_{1} \delta_{c}-Y_{c} \tilde{\alpha}_{c}-\operatorname{sgn}\left(\delta_{c}\right)\left\|\delta_{n}\right\||\eta|-K_{2} \operatorname{sgn}\left(\delta_{c}\right)\left\|\delta_{n}\right\|-\xi \\
W^{T} \phi+\varepsilon
\end{array}\right] } \\
& -\delta^{T} \tau_{d}+\dot{\hat{\alpha}}_{c}^{T} \Gamma^{-1} \tilde{\alpha}_{c}+\operatorname{tr}\left\{\tilde{W}^{T} \Upsilon^{-1} \dot{\tilde{W}}\right\} \\
& +\left(\kappa-\varepsilon_{T}\right) \dot{\kappa}+\eta^{\frac{-1}{2 n+1}} \dot{\eta} \\
& =-\delta_{c}^{T} K_{1} \delta_{c}-\left\|\delta_{c}\right\|\left\|\delta_{n}\right\||\eta|-\delta_{c}^{T} \xi+\delta_{n}^{T}\left(\varepsilon-\tau_{d n}\right)
\end{aligned}
$$




$$
\begin{aligned}
& -K_{2}\left\|\delta_{c}\right\|\left\|\delta_{n}\right\|-\delta_{c}^{T} \tau_{\mathrm{dc}}-\frac{\left\|\delta_{n}\right\|^{2}}{\left\|\delta_{n}\right\|+\mu} \kappa-K_{3}\left\|\delta_{n}\right\|^{2} \\
& +\operatorname{tr}\left\{\tilde{W}^{T} \Upsilon^{-1}\left(\dot{\tilde{W}}+\Upsilon \delta_{n}^{T} \phi\right)\right\} \\
& +\left(\kappa-\varepsilon_{T}\right) \dot{\kappa}-\tilde{\alpha}_{c}^{T} \Gamma^{\prime} \Gamma^{-1} \tilde{\alpha}_{c} \\
& \leq-\delta_{c}^{T} K_{1} \delta_{c}-\left\|\delta_{c}\right\|\left\|\delta_{n}\right\||\eta|-\delta_{c}^{T} \xi+\left\|\delta_{n}\right\| \varepsilon_{T} \\
& -K_{2}\left\|\delta_{c}\right\|\left\|\delta_{n}\right\|-\delta_{c}^{T} \tau_{\mathrm{dc}}-\frac{\left\|\delta_{n}\right\|^{2}}{\left\|\delta_{n}\right\|+\mu} \kappa \\
& -K_{3}\left\|\delta_{n}\right\|^{2}+\left(\kappa-\varepsilon_{T}\right) \dot{\kappa}+t r\left\{\tilde{W}^{T} \Upsilon^{-1}\left(\dot{\tilde{W}}+\Upsilon \delta_{n}^{T} \phi\right)\right\} \\
& -\tilde{\alpha}_{c}^{T} \Gamma^{\prime} \Gamma^{-1} \tilde{\alpha}_{c} \\
& \leq-\delta_{c}^{T} K_{1} \delta_{c}-\left\|\delta_{c}\right\|\left\|\delta_{n}\right\||\eta|-\delta_{c}^{T} \xi-\delta_{c}^{T} \tau_{\mathrm{dc}} \\
& -K_{2}\left\|\delta_{c}\right\|\left\|\delta_{n}\right\|-K_{3}\left\|\delta_{n}\right\|^{2}+\mu \varepsilon_{T}-\tilde{\alpha}_{c}^{T} \Gamma^{\prime} \Gamma^{-1} \tilde{\alpha}_{c} \\
& \dot{V} \leq-K_{1}\left\|\delta_{c}\right\|^{2}-K_{3}\left\|\delta_{n}\right\|^{2}-\Gamma^{\prime} \Gamma^{-1}\left\|\tilde{\alpha}_{c}\right\|^{2} \\
& -\delta_{c}^{T} \xi-\delta_{c}^{T} \tau_{\mathrm{dc}}+\mu \varepsilon_{T} \\
& \quad=-\left\|\delta^{\prime}\right\|^{T} K^{\prime}\left\|\delta^{\prime}\right\|-\delta_{c}^{T} \xi-\delta_{c}^{T} \tau_{\mathrm{dc}}+\mu \varepsilon_{T}
\end{aligned}
$$

where $K^{\prime}=\operatorname{diag}\left[K_{1}, K_{3}, \Gamma^{\prime} \Gamma^{-1}\right]$ and $\delta^{\prime}=\left[\delta_{c}, \delta_{n}\right.$, $\left.\tilde{\alpha}_{c}\right]^{T}$.

Considering that $\Gamma^{\prime}$ and $\Gamma^{-1}$ are positive definite diagonal matrix, thus $\Gamma^{\prime} \Gamma^{-1}$ is a positive definite diagonal matrix.

Case 1. For the case when $\tau_{\mathrm{dc}}=0$, design the collocated robust compensator as $\xi=0$ and integrate both sides of (36) from $t=0$ to $t=T$ as

$V(T)-V(0) \leq-\int_{0}^{T}\left\|\delta^{\prime}\right\|^{T} K^{\prime}\left\|\delta^{\prime}\right\| \mathrm{d} t+\varepsilon_{T} \int_{0}^{T} \mu \mathrm{d} t$

Considering that $V(T) \geq 0$ and $\int_{0}^{\infty} \mu \mathrm{d} t<\infty$, we have

$$
\begin{aligned}
& \lim _{T \rightarrow \infty} \sup \frac{1}{T} \int_{0}^{T}\left\|\delta^{\prime 2}\right\| \mathrm{d} t \\
& \quad \leq \frac{1}{K^{\prime}}\left(V(0)+\varepsilon_{T} \int_{0}^{T} \mu \mathrm{d} t\right) \lim _{T \rightarrow \infty} \frac{1}{T}
\end{aligned}
$$

Case 2. For the case when $\tau_{\mathrm{dc}} \neq 0$ and $\left\|\tau_{\mathrm{dc}}\right\|<\beta_{m}$, the collocated robust compensator $\xi$ is designed to satisfy the following conditions

$$
\left\{\begin{array}{l}
\delta_{c}^{T} \xi \geq 0 \\
\beta_{m}\left\|\delta_{c}\right\|-\delta_{c}^{T} \xi \leq \rho
\end{array}\right.
$$

where $\beta_{m}$ is the upper bound of $\tau_{\mathrm{dc}}$ and $\rho$ is a positive design scalar.

Theorem 2 Consider following control laws to the uncertain underactuated system

$$
\begin{aligned}
\tau= & \tau_{c}+\tau_{n} \\
\tau_{c}= & Y_{c} \hat{\alpha}_{c}-K_{1} \delta_{c}-\xi, \tau_{n}=-\operatorname{sgn}\left(\delta_{c}\right)\left\|\delta_{n}\right\||\eta| \\
& -K_{2} \operatorname{sgn}\left(\delta_{c}\right)\left\|\delta_{n}\right\|
\end{aligned}
$$

with the Adaptation Algorithm 2 designed in (34), and the collocated robust compensator $\xi$ designed using hyperbolic tangent function as

$\xi=\beta_{m} \tanh \left(\frac{n \eta_{r} \beta_{m} \delta_{c}}{\rho}\right)$

with $\eta_{r}$ is a gain constant chosen as $\eta_{r}=0.2785$ here, and the auxiliary input $\eta$ in (40b) is constructed as

$\dot{\eta}=\eta^{\frac{1}{2 n+1}}\left(-K_{3}\left\|\delta_{n}\right\|^{2}-\left\|\delta_{n}\right\| \hat{W}^{T} \phi\right)$

with the adaptation law for $\hat{W}$ based on the projection algorithm, given by

$$
\begin{aligned}
\dot{\hat{W}}= & -\dot{\tilde{W}} \\
= & \left\{\begin{array}{l}
\Upsilon \phi \delta_{n}^{T}-\beta \Upsilon\left\|\delta_{n}\right\| \hat{W}-\frac{\delta_{n}^{T} \hat{W}^{T} \Upsilon \phi \hat{W}}{W_{N}}, \\
\quad \text { if } \operatorname{tr}\left\{\hat{W}^{T} \hat{W}\right\}=W_{N} \text { and } \delta_{n}^{T} \hat{W}^{T} \phi \leq 0 ; \\
\Upsilon \phi \delta_{n}^{T}-\beta \Upsilon\left\|\delta_{n}\right\| \hat{W}, \quad \text { iftr }\left\{\hat{W}^{T} \hat{W}\right\} \quad(40 \mathrm{e}) \\
\quad<W_{N} \text { or if } \operatorname{tr}\left\{\hat{W}^{T} \hat{W}\right\} \\
=W_{N} \text { and } \delta_{n}^{T} \hat{W}^{T} \phi>0 .
\end{array}\right.
\end{aligned}
$$

Then, it follows:

(1) $\operatorname{tr}\left\{\hat{W}^{T} \hat{W}\right\} \leq W_{N}$ holds.

(2) All signals in the collocated and non-collocated systems are UUB.

Proof Consider the Lyapunov function as follows

$$
\begin{aligned}
V= & \frac{1}{2} \delta^{T} D \delta+\frac{1}{2} \tilde{\alpha}_{c}^{T} \Gamma^{-1} \tilde{\alpha}_{c} \\
& +\frac{1}{2} \operatorname{tr}\left\{\tilde{W}^{T} \Upsilon^{-1} \tilde{W}\right\}+\frac{2 n+1}{2 n} \eta^{\frac{2 n}{2 n+1}}
\end{aligned}
$$

The derivative of Lyapunov candidate function is yielded as 


$$
\begin{aligned}
\dot{V}= & {\left[\delta_{c}^{T} \delta_{n}^{T}\right] } \\
& {\left[\begin{array}{c}
\left.-K_{1} \delta_{c}-Y_{c} \tilde{\alpha}_{c}-\operatorname{sgn}\left(\delta_{c}\right)\left\|\delta_{n}\right\||\eta|-K_{2} \operatorname{sgn}\left(\delta_{c}\right)\left\|\delta_{n}\right\|-\xi\right] \\
W^{T} \phi+\varepsilon
\end{array}\right] } \\
& -\delta^{T} \tau_{d}+\dot{\hat{\alpha}}_{c}^{T} \Gamma^{-1} \tilde{\alpha}_{c}+\operatorname{tr}\left\{\tilde{W}^{T} \Upsilon^{-1} \dot{\tilde{W}}\right\}+\eta^{\frac{-1}{2 n+1}} \dot{\eta} \\
= & -\left\|\delta_{c}\right\|\left\|\delta_{n}\right\||\eta|-\delta_{c}^{T} K_{1} \delta_{c}-K_{2}\left\|\delta_{c}\right\|\left\|\delta_{n}\right\| \\
& -\delta_{c}^{T} \xi-\delta_{c}^{T} \tau_{\mathrm{dc}}-K_{3}\left\|\delta_{n}\right\|^{2} \\
& +\operatorname{tr}\left\{\tilde{W}^{T} \Upsilon^{-1}\left(\dot{\tilde{W}}+\Upsilon \delta_{n}^{T} \phi\right)\right\} \\
& +\delta_{n}^{T}\left(\varepsilon-\tau_{d n}\right)-\tilde{\alpha}_{c}^{T} \Gamma^{\prime} \Gamma^{-1} \tilde{\alpha}_{c} \\
& \leq-\left\|\delta_{c}\right\|\left\|\delta_{n}\right\||\eta|-K_{2}\left\|\delta_{c}\right\|\left\|\delta_{n}\right\|-\delta_{c}^{T} K_{1} \delta_{c}-\delta_{c}^{T} \xi \\
& +-K_{3}\left\|\delta_{n}\right\|^{2} \\
& +t r\left\{\tilde{W}^{T} \Upsilon^{-1}\left(-\Upsilon \phi \delta_{n}^{T}+\beta \Upsilon\left\|\delta_{n}\right\| \hat{W}+\Upsilon \delta_{n}^{T} \phi\right)\right\} \\
& \left\|\delta_{n}\right\| \varepsilon_{T}-\delta_{c}^{T} \tau_{\mathrm{dc}}-\tilde{\alpha}_{c}^{T} \Gamma^{\prime} \Gamma^{-1} \tilde{\alpha}_{c} \\
\dot{V} \leq & -\delta_{c}^{T} K_{1} \delta_{c}-\Gamma^{\prime} \Gamma^{-1}\left\|\tilde{\alpha}_{c}\right\|^{2}-K_{3}\left\|\delta_{n}\right\|^{2}-\delta_{c}^{T} \xi \\
& -\delta_{c}^{T} \tau_{\mathrm{dc}}+\beta\left\|\delta_{n}\right\| \operatorname{tr}\left\{\tilde{W}^{T}(W-\tilde{W})\right\}+\delta_{n}^{T} \varepsilon_{T}
\end{aligned}
$$

Let us decompose (42) into the following two parts

$$
\begin{aligned}
& \dot{V}_{1}=-K_{3}\left\|\delta_{n}\right\|^{2}+\beta\left\|\delta_{n}\right\| \operatorname{tr}\left\{\tilde{W}^{T}(W-\tilde{W})\right\}+\delta_{n}^{T} \varepsilon_{T} \\
& \dot{V}_{2}=-\Gamma^{\prime} \Gamma^{-1}\left\|\tilde{\alpha}_{c}\right\|^{2}-\delta_{c}^{T} K_{1} \delta_{c}-\delta_{c}^{T} \tau_{\mathrm{dc}}-\delta_{c}^{T} \xi
\end{aligned}
$$

We have

(1) For $\dot{V}_{1}$, considering that

$$
\begin{aligned}
\operatorname{tr}\left\{\tilde{W}^{T}(W-\tilde{W})\right\} & =(\tilde{W}, W)_{F}-\|\tilde{W}\|_{F}^{2} \\
& \leq\|\tilde{W}\|_{F}\|W\|_{F}-\|\tilde{W}\|_{F}^{2}
\end{aligned}
$$

Substituting (44) into (43a), we have

$$
\begin{aligned}
\dot{V}_{1} \leq & -\lambda_{\min }\left(K_{3}\right)\left\|\delta_{n}\right\|^{2} \\
& +\beta\left\|\delta_{n}\right\|\|\tilde{W}\|_{F}\left(W_{\max }-\|\tilde{W}\|_{F}\right)+\varepsilon_{T}\left\|\delta_{n}\right\| \\
= & -\left\|\delta_{n}\right\|\left(\lambda_{\min }\left(K_{3}\right)\left\|\delta_{n}\right\|\right. \\
& \left.+\beta\|\tilde{W}\|_{F}\left(\|\tilde{W}\|_{F}-W_{\max }\right)-\varepsilon_{T}\right)
\end{aligned}
$$

Since

$$
\begin{aligned}
\lambda_{\min }\left(K_{3}\right)\left\|\delta_{n}\right\|+\beta\|\tilde{W}\|_{F}\left(\|\tilde{W}\|_{F}-W_{\max }\right)-\varepsilon_{T} \\
=\beta\left(\|\tilde{W}\|_{F}-\frac{W_{\max }}{2}\right)^{2}-\beta \frac{W_{\max }^{2}}{4} \\
+\lambda_{\min }\left(K_{3}\right)\left\|\delta_{n}\right\|-\varepsilon_{T}
\end{aligned}
$$

To guarantee $\dot{V}_{1} \leq 0$, the following inequality needs to be satisfied

$$
\begin{aligned}
\left\|\delta_{n}\right\| & >\frac{\beta W_{\max }^{2}+4 \varepsilon_{T}}{4 \lambda_{\min }\left(K_{3}\right)} \text { or }\|\tilde{W}\|_{F} \\
& >\frac{W_{\max }}{2}+\sqrt{\frac{W_{\max }^{2}}{4}+\frac{\varepsilon_{T}}{\beta}}
\end{aligned}
$$

Therefore, $\dot{V}_{1}$ is negative outside a compact set. Based on the standard Lyapunov theorem extension, the UUB of both $\delta_{n}$ and $\|\tilde{W}\|_{F}$ is demonstrated.

Through (43b), the time derivative of $V_{2}$ can be given by

$$
\dot{V}_{2} \leq-\Gamma^{\prime} \Gamma^{-1}\left\|\tilde{\alpha}_{c}\right\|^{2}-\delta_{c}^{T} K_{1} \delta_{c}+\beta_{m}\left\|\delta_{c}\right\|-\delta_{c}^{T} \xi
$$

Based on the above knowledge of the design requirement (39), the definition of $V$ and $\dot{V}_{2}$, as well as the assumption of boundedness of neural network weight, we substitute the collocated robust compensator (40c) into (43b) and yield

$$
\begin{aligned}
\dot{V}_{2} & \leq-\Gamma^{\prime} \Gamma^{-1}\left\|\tilde{\alpha}_{c}\right\|^{2}-\delta_{c}^{T} K_{1} \delta_{c}+\rho \\
& =-\vartheta^{T} K_{4} \vartheta+\rho \\
& \leq-\lambda_{\min }\left(K_{4}\right)\|\vartheta\|^{2}+\rho
\end{aligned}
$$

where $K_{4}=\operatorname{diag}\left\{\Gamma^{\prime} \Gamma^{-1}, K_{1}\right\}$ and $\lambda_{\min }\left(K_{4}\right)$ is the minimum eigenvalue of the matrix $K_{4}$. As a result, $\dot{V}_{2}$ is strictly negative outside the following compact set $\Sigma_{\vartheta}$ :

$\Sigma_{\vartheta}=\left\{\vartheta(t) \mid 0 \leq\|\vartheta\| \leq \sqrt{\frac{\rho}{\lambda_{\min }\left(K_{4}\right)}}\right\}$

Therefore, it is concluded that the filtered tracking error $\delta_{c}$ for collocated subsystem and $\delta_{n}$ for non-collocated subsystem, and the estimation error $\tilde{W}$ of the parameters are uniformly ultimately bounded. The tracking error of collocated subsystem decreases whenever $\vartheta$ is outside the compact set $\Sigma_{\vartheta}$, and thus $\|\vartheta\|$ is UUB. Considering that all the signals included in the control system (40) are UUB, it is therefore concluded that the control system (40) is uniformly ultimately bounded. 


\section{Simulations}

In this section, simulation studies are conducted to demonstrate the effectiveness of the designed control schemes from the examples of an 2-DOF underactuated manipulator and 2-DOF vibro-driven system [5]. The consideration behind is to investigate the effectiveness towards both underactuated manipulation and locomotion systems.

\subsection{2-DOF underactuated manipulator}

The two-link planar manipulator as shown in Fig. 2 has its first link actuated and the second link unactuated. Two revolute joints are used to connect link 1 and link 2 , and in the horizontal plane, link 1 is able to rotate 360 degrees. The denotations are introduced as follows: for link $i, i=1,2, q_{i}$ are the generalized coordinate and the joint angle of each link, $m_{i}$ and $l_{i}$ are the mass and length, respectively. $l_{c i}$ represents the length from the previous joint to the COM of link $i$, and $I_{i}$ is the moment of inertia about the axis coming out of the page and coming through the COM of link $i$.

The equations of motion of the manipulator can be derived using the Lagrange's approach as follows:

$D(q) \ddot{q}+C(q, \dot{q}) \dot{q}+G(q)+F_{v} \dot{q}+F_{c}(q, \dot{q})+\tau_{d}=\tau$

where

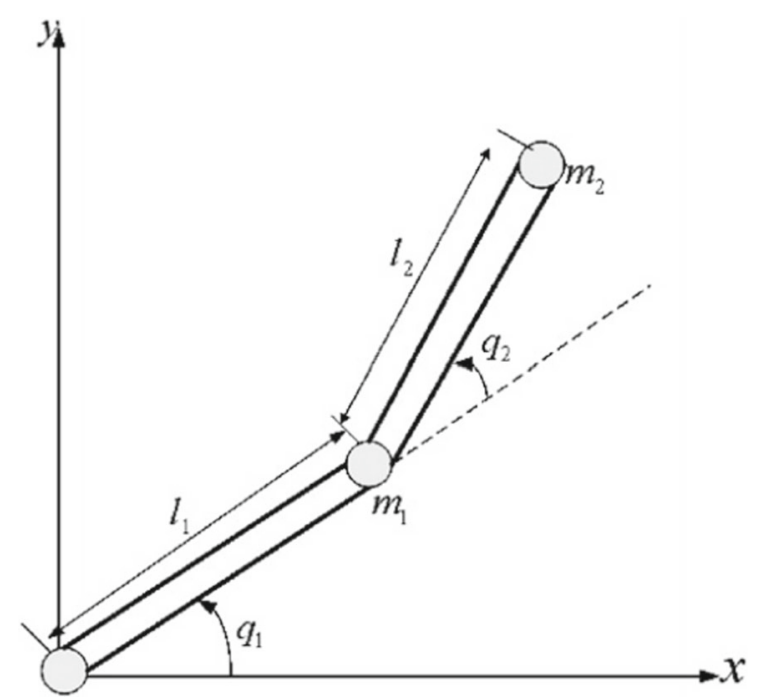

Fig. 2 The 2-DOF underactuated manipulator

eter is $\alpha=\left[\alpha_{1} \alpha_{2} \alpha_{3} \alpha_{4} \alpha_{5}\right]^{T} \in R^{5}$. Based on the auxiliary kinematic vector variables defined in (20), the collocated regressor $Y_{c}$ is therefore obtained as $Y_{c}=\left[-\dot{\varrho}_{1} Y_{c 2}-\dot{\varrho}_{2}-g \cos q_{1}-g \cos \left(q_{1}+q_{2}\right)\right]$ with $Y_{c 2}=-\left(2 \cos q_{2} \dot{\varrho}_{1}+\cos q_{2} \dot{\varrho}_{2}-\dot{q}_{2} \varrho_{1} \sin q_{2}-\sin q_{2}\left(\dot{q}_{1}+\right.\right.$ $\left.\left.\dot{q}_{2}\right) \varrho_{2}\right)$.

Generically, the adaptive NN-based tracking control scheme in (40) is evaluated with matched and mismatched uncertainties. The rationality of system parameter values selection of the manipulator in this section is configured from studies in the literature as reported in [45] as follows: $m_{1}=m_{2}=2 \mathrm{Kg}$, $I_{1}=I_{2}=0.2528 \mathrm{Kgm}^{2}, l_{c 1}=l_{c 2}=0.75 \mathrm{~m}$, $l_{1}=l_{2}=1.5 \mathrm{~m}$. The initial conditions are set as

$$
\begin{aligned}
& D(q)=\left[\begin{array}{cc}
m_{2}\left(l_{1}^{2}+l_{c 2}^{2}+2 l_{1} l_{c 2} \cos q_{2}\right)+m_{1} l_{c 1}^{2}+I_{1}+I_{2} & m_{2}\left(l_{1} l_{c 2} \cos q_{2}+l_{c 2}^{2}\right)+I_{2} \\
m_{2}\left(l_{1} l_{c 2} \cos q_{2}+l_{c 2}^{2}\right)+I_{2} & I_{2}+m_{2} l_{c 2}^{2}
\end{array}\right], \\
& C(q, \dot{q})=\left[\begin{array}{cc}
-m_{2} l_{c 2} l_{1} \sin q_{2} \dot{q}_{2}-m_{2} l_{c 2} l_{1} \sin q_{2}\left(\dot{q}_{1}+\dot{q}_{2}\right) \\
m_{2} l_{c 2} l_{1} \sin q_{2} \dot{q}_{1}
\end{array}\right], \\
& G(q)=\left[\begin{array}{c}
\left(m_{2} l_{1}+m_{1} l_{c 1}\right) g \cos q_{1}+m_{2} l_{c 2} g \cos \left(q_{1}+q_{2}\right) \\
m_{2} l_{c 2} g \cos \left(q_{1}+q_{2}\right)
\end{array}\right], \\
& F_{v} \dot{q}+F_{c}(q, \dot{q})=\left[\begin{array}{c}
f_{v 1} \dot{q}_{1}+c_{1} \operatorname{sgn}\left(\dot{q}_{1}\right) \\
f_{v 2} \dot{q}_{2}+c_{2} \operatorname{sgn}\left(\dot{q}_{2}\right)
\end{array}\right], \tau_{d}=\left[\begin{array}{l}
a_{1} \sin (t) \\
a_{2} \sin (t)
\end{array}\right], \tau=\left[\begin{array}{l}
\tau_{1} \\
0
\end{array}\right] .
\end{aligned}
$$

It is assumed that the moments of inertia are calculated in the form of $I_{i}=\frac{m_{i} l_{i}^{2}}{12}$. The unknown parameters are chosen as $\alpha_{1}=m_{2}\left(l_{1}^{2}+l_{c 2}^{2}\right)+m_{1} l_{c 1}^{2}+I_{1}+I_{2}$, $\alpha_{2}=m_{2} l_{c 2} l_{1}, \alpha_{3}=I_{2}+m_{2} l_{c 2}^{2}, \alpha_{4}=m_{2} l_{1}+$ $m_{1} l_{c 1}, \alpha_{5}=m_{2} l_{c 2}$, and then the uncertain param- $q(0)=\left[q_{1}(0) q_{2}(0)\right]^{\mathrm{T}}=[0.09-0.09]^{\mathrm{T}}, \dot{q}(0)=$ $\left[\dot{q}_{1}(0) \dot{q}_{2}(0)\right]^{\mathrm{T}}=[00]^{\mathrm{T}}$, and the reference trajectory is given as $q_{1 d}(t)=0.5 \pi(1+\sin (0.1 t))$ [45]. It is noted that when the desired trajectory for $q_{1}$ is chosen, the prior knowledge of the desired trajectory for 


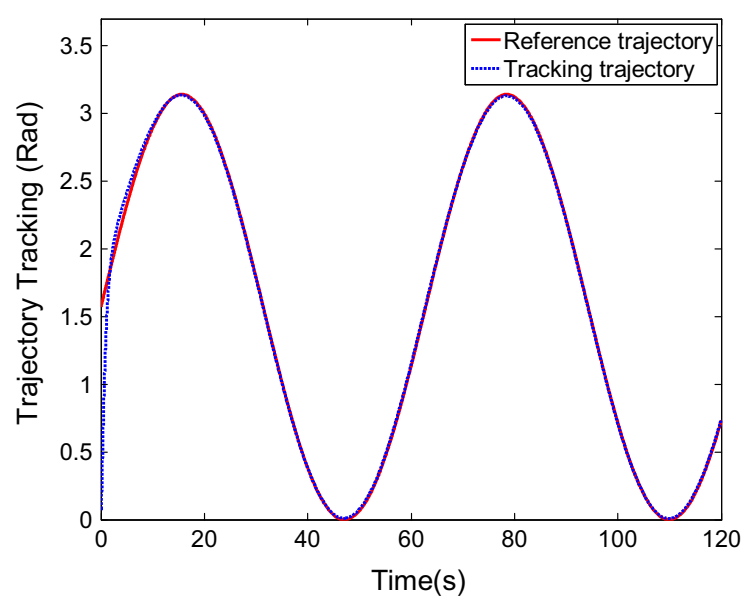

Fig. 3 Trajectory tracking performance

$q_{2}$ can be achieved through convenient computation, and it should satisfy the following constraint equation

$$
\begin{gathered}
D_{21} \ddot{q}_{1}+D_{22} \ddot{q}_{2}+C_{21} \dot{q}_{1}+C_{22} \dot{q}_{2} \\
+G_{2}+F_{v 1} \dot{q}+F_{c 1}+\tau_{d 1}=0
\end{gathered}
$$

The parameter values of friction and disturbance are chosen as $c_{1}=c_{2}=0.02, a_{1}=a_{2}=0.2$. The bandwidth of the first-order filter is set as $\Lambda=\left[\Lambda_{1} \Lambda_{2}\right]^{\mathrm{T}}=$ $[1230]^{\mathrm{T}}$. In the simulation, parameters of the control schemes are chosen to be $K_{1}=2 I, K_{2}=5 I$ and $K_{3}=20 I$. The adaptation gains are chosen as $\Gamma^{\prime}=8 I$ and $\Gamma=4 I$. Parameter values for the collocated robust compensator are set as $\beta_{m}=20, \rho=0.5$. In addition, the weight tuning parameter of the designed control schemes is chosen as $\Upsilon=0.005$ and $\beta=0.1$. The rationality of these selections is configured using iterative simulations.

Simulation results of the trajectory tracking performance of the adaptive NN-based control system (40) are presented in Fig. 3 with time-varying matched and mismatched disturbances. The reference trajectory (red solid line), the tracking trajectory (blue dashed line) in Fig. 3, the trajectory tracking error in Fig. 4, the control torque in Fig. 5 and the NN approximation performance in Figs. 6 and 7 are portrayed. We can see that the proposed scheme demonstrates good performance under the model uncertainties, frictions and time-varying external disturbances. It can be observed from Fig. 4 that the system tracks the reference trajectory accurately and the tracking error converges to a small compact set after about $4 \mathrm{~s}$. The bounded control

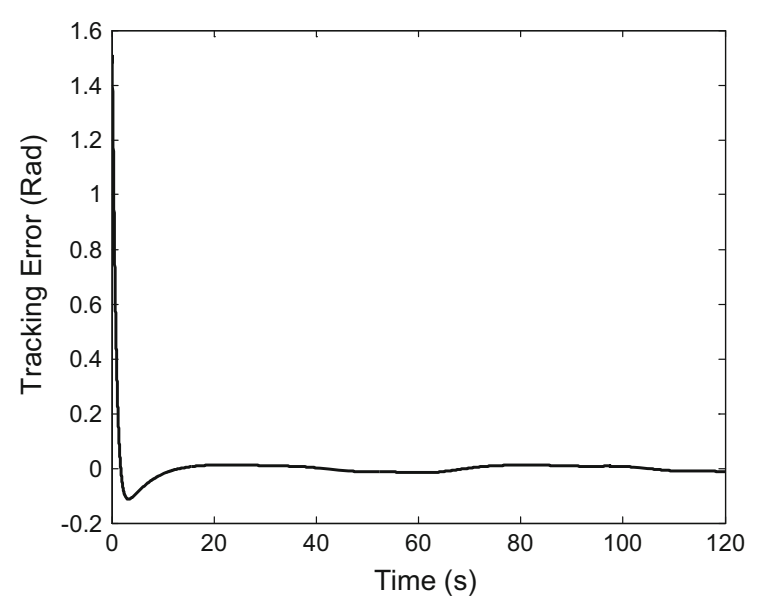

Fig. 4 Trajectory tracking error

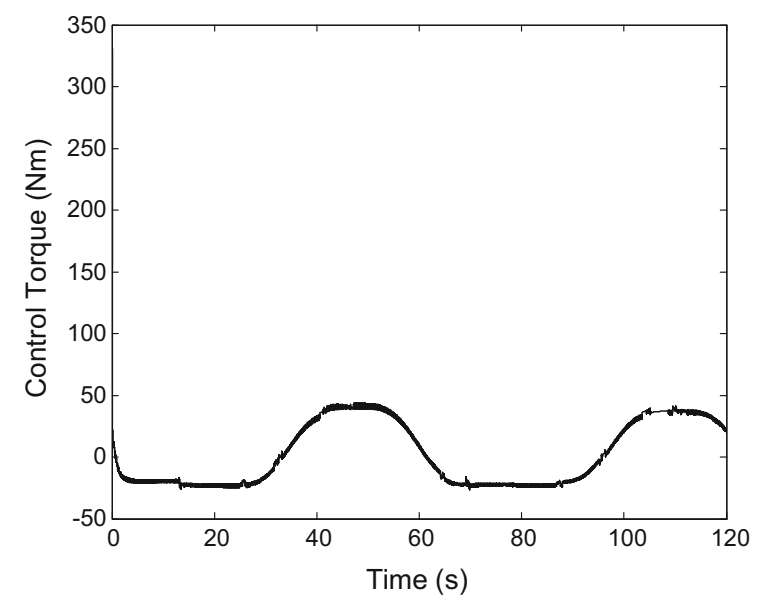

Fig. 5 Control input torque

input torque by using the designed control scheme is shown in Fig. 5 with an upper bound of $50 \mathrm{Nm}$ and a lower bound of $-20 \mathrm{Nm}$. The RBFNN approximates the nonlinear uncertainties $\chi(\mathrm{z})$ effectively from Fig. 7 . From the simulation studies, we can draw a conclusion that the developed control system is able to adapt the model uncertainties and is robust against the matched and mismatched external disturbances.

\subsection{2-DOF underactuated vibro-driven system}

The simulation study in Sect. 4.1 considers an underactuated manipulator with its base mounted on the working surface under uncertain dynamics and external disturbances. The passive dynamics of the second link 


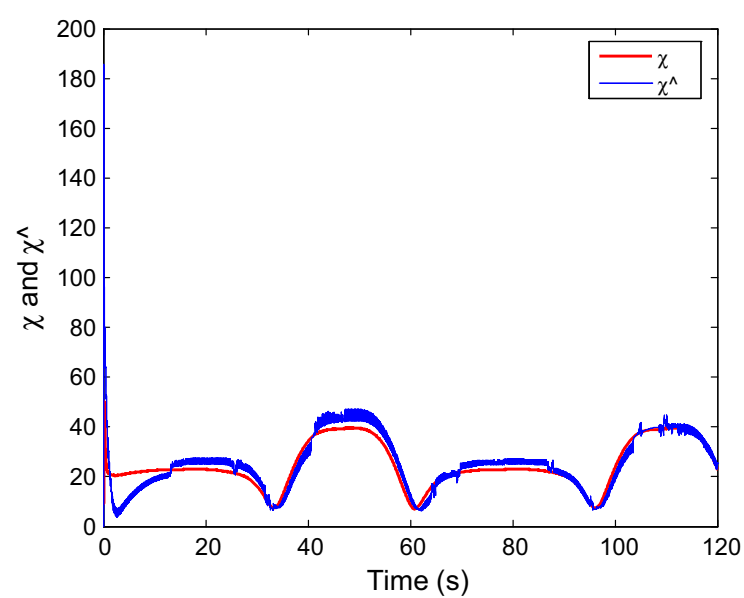

Fig. $6 x(z)$ and $\hat{x}(z)$

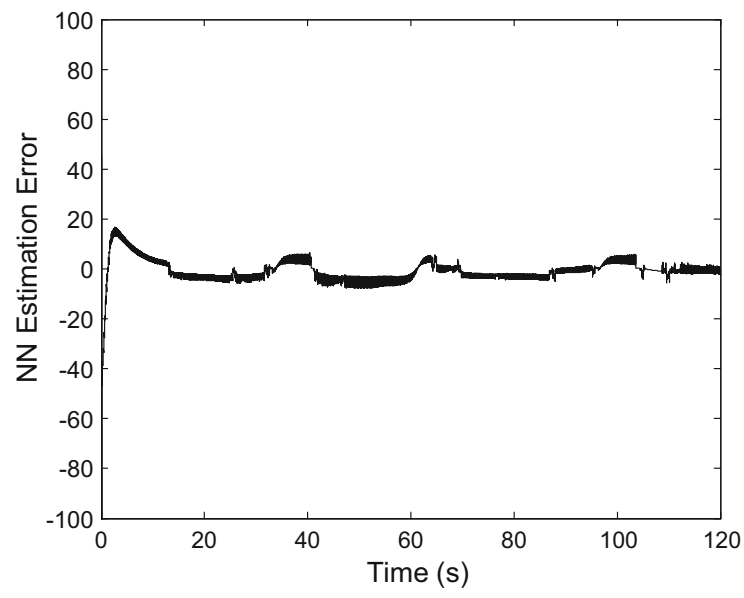

Fig. $7 \mathrm{NN}$ approximation error

and the actuated dynamics of the first link are coupled; thus, the unmodelled dynamics of the second link may contribute additional time-varying inertia and nonlinearity to the manipulator dynamics. In this subsection, the context of an underactuated mobile robotic model is considered as shown in Fig. 8. This underactuated vibro-driven robotic system was proposed in [3] for which the actuated and unactuated dynamics are strongly coupled.

In the presence of matched and mismatched external disturbances, the underactuated dynamics of the vibrodriven system are given as

$D(q) \ddot{q}+C(q, \dot{q}) \dot{q}+K(q) q+G(q)+F+\tau_{d}=B \tau$

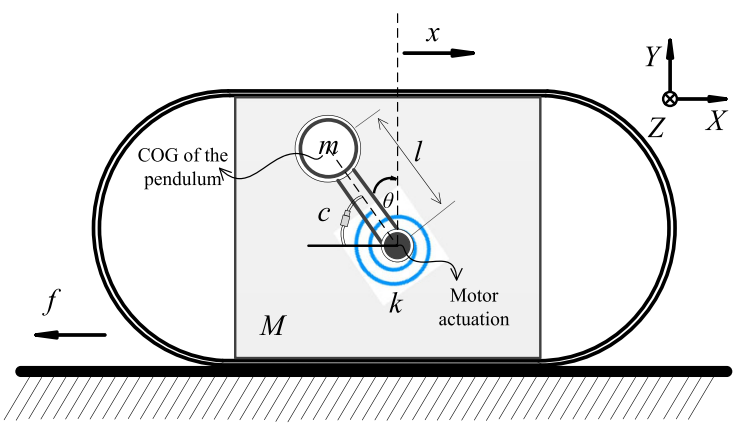

Fig. 8 Schematic of the underactuated vibro-driven system

where $D(q)=\left[\begin{array}{cc}m l^{2} & -m l c_{\theta} \\ -m l c_{\theta} & (M+m)\end{array}\right]$ denotes the inertia matrix, $C(q, \dot{q})=\left[\begin{array}{cc}0 & 0 \\ m l_{s_{\theta}} \dot{\theta} & 0\end{array}\right]$ is the Centripetal and Coriolis matrix, $K(q)=\left[\begin{array}{ll}k & 0 \\ 0 & 0\end{array}\right]$ represents the generalized stiffness matrix, $G(q)=\left[-m g l s_{\theta} 0\right]^{T}$ represents the gravitational torques, $F=[c \dot{\theta} f]^{T}$ is the friction forces, $\tau_{d}=\left[\tau_{\mathrm{dc}} \tau_{d n}\right]^{T}=\left[\begin{array}{l}a_{1} \sin (t) \\ a_{2} \sin (t)\end{array}\right]$ denotes the external matched and mismatched disturbances, $B=[10]^{T}$ is the input force matrix and $\tau \in \mathcal{R}^{1}$ denotes the control input applied to the system.

In the simulation, the rationality of the parameter values selection in this section is specified as follows: the system parameter values are configured from the studies in literature as reported in $[48,49]$ as $M=$ $0.5 \mathrm{~kg}, m=0.138 \mathrm{~kg}, l=0.3 \mathrm{~m}, g=9.81 \mathrm{~m} / \mathrm{s}^{2}$, $\mu=0.01 \mathrm{~N} / \mathrm{ms}$. Initial conditions of the system are set as $\theta(0)=\theta_{0}=\pi / 3, \dot{\theta}(0)=0, x(0)=0$ and $\dot{x}(0)=0$. The simulation is conducted in $6.6 \mathrm{~s}$ which is one full motion cycle. The parameter values for the matched and mismatched external disturbances are chosen as $a_{1}=a_{2}=0.2$. The bandwidth of the firstorder filter is set as $\Lambda=\left[\Lambda_{1} \Lambda_{2}\right]^{T}=[1520]^{T}$. In the simulation, the controller parameters are chosen to be $K_{1}=10 I, K_{2}=20 I$ and $K_{3}=50 I$. The adaptation gains are chosen as $\Gamma^{\prime}=10 I$ and $\Gamma=6 I$. Parameter values for the collocated robust compensator are set as $\beta_{m}=20, \rho=0.5$. In addition, the weight tuning parameter of the proposed control system is selected as $\Upsilon=0.005$ and $\beta=0.1$. The rationality of these selections is configured using iterative simulations.

The trajectory tracking performance of the actuated subsystem is presented in Fig. 9. It is observed from the figure that although the response of the proposed 


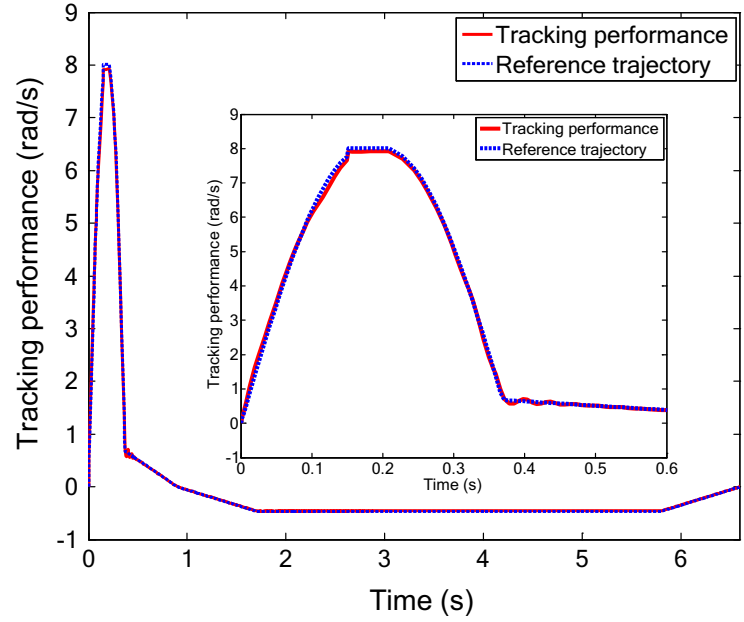

Fig. 9 Trajectory tracking performance

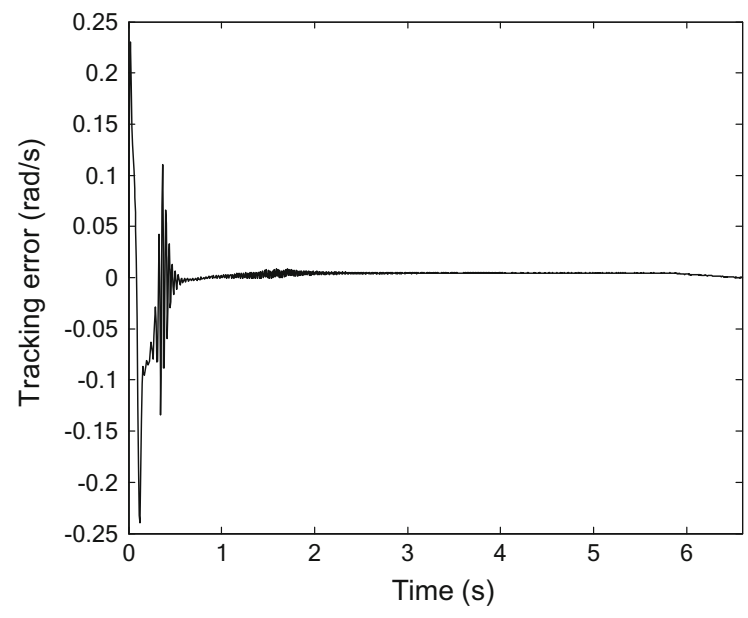

Fig. 10 Trajectory tracking error

control scheme is slightly slower, the controlled pendulum trajectory tracks the reference trajectory accurately. The reference trajectory for the actuated subsystem is chosen as shown in Fig. 9. The proposed control system has a learning process that makes the estimated parameters adapt to appropriate values.

The tracking error is shown in Fig. 10, from which the tracking error converges to an adjacent and bounded compact set near zero in finite time. The trajectory of the vibro-driven system is presented in Fig. 11 showing that the cart travels at the speed about $7 \mathrm{~cm}$ within $6.6 \mathrm{~s}$. The control torque is shown in Fig. 12 that demonstrates the boundedness of the torque input. As demonstrated in the system performance, the developed $\mathrm{NN}$ adaptive

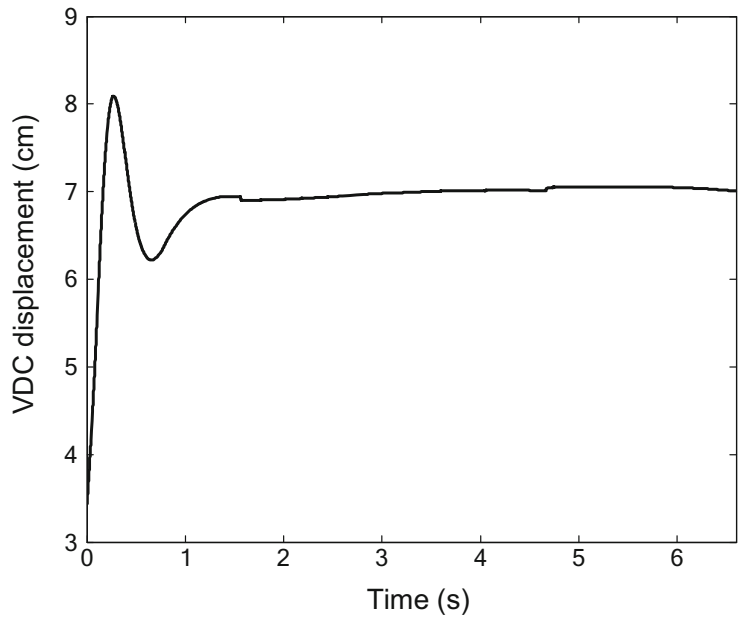

Fig. 11 Performance of the vibro-driven system

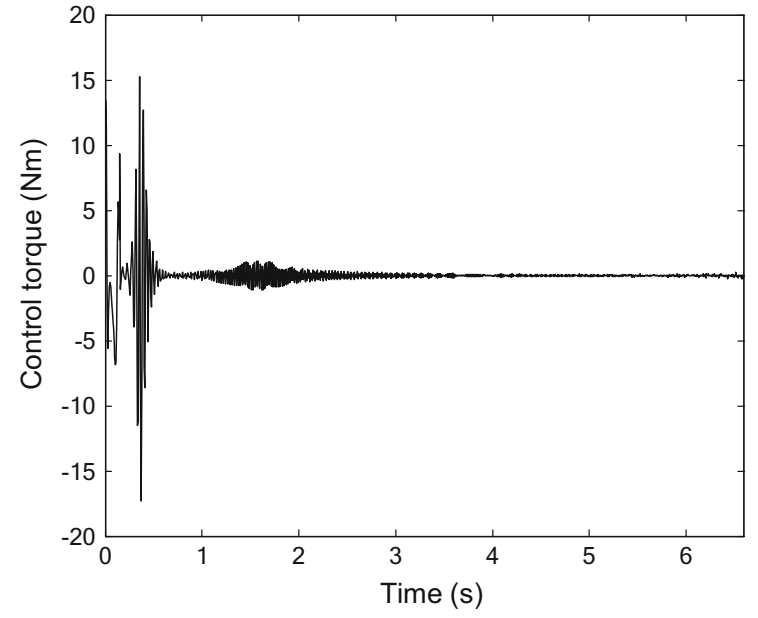

Fig. 12 Control torque

control scheme is capable of guaranteeing accurate trajectory tracking of the actuated subsystem, and meanwhile, the passive subsystem can maintain a forward locomotion at some desired velocity. Therefore, it is concluded that the designed control system is efficient in the presence of unknown nonlinear dynamic systems and environmental disturbances.

\section{Conclusions}

In this paper, novel NNs-based adaptive tracking control schemes for underactuated systems with matched and mismatched disturbances have been presented. The 
parametric uncertainties and matched and mismatched external disturbances have been considered in the controller design, which feature a generic model in the research of underactuated systems. The mismatched disturbances have been omitted in most of the existing approaches for the tracking control of UMSs. Auxiliary control variables have been designed to establish the controllability of non-collocated subset of underactuated systems by using a universal approximation of RBFNN, and approximation errors and external disturbances can be efficiently counteracted through design of robust compensators. Employing the adaptive control approach, combined with variable structure and NNs, the exact values of the parameters of the underactuated systems are not required to be known a priori. The stability of the overall system has been proved by Lyapunov analysis, and it is shown that the tracking error can be reduced as small as desired in finite time by choosing appropriate controller parameters. The simulation studies on an underactuated manipulator and an underactuated vibro-driven system have shown the effectiveness of the proposed adaptive control systems.

Acknowledgements This work was partially supported by the National Natural Science Foundation of China (61803396), European Commission Marie Skłodowska-Curie SMOOTH (smart robots for fire-fighting) project (H2020-MSCA-RISE2016-734875) and Royal Society International Exchanges Scheme (Adaptive Learning Control of a Cardiovascular Robot using Expert Surgeon Techniques) project (IE151224). FP7 People: Marie-Curie Actions (Grant Number PIRSES-GA-2012318902).

\section{Compliance with ethical standards}

Conflicts of interest The authors declare that they have no conflict of interest.

Open Access This article is distributed under the terms of the Creative Commons Attribution 4.0 International License (http:// creativecommons.org/licenses/by/4.0/), which permits unrestricted use, distribution, and reproduction in any medium, provided you give appropriate credit to the original author(s) and the source, provide a link to the Creative Commons license, and indicate if changes were made.

\section{References}

1. Azimi, M.M., Koofigar, H.R.: Adaptive fuzzy backstepping controller design for uncertain underactuated robotic systems. Nonlinear Dyn. 79, 1457-1468 (2015). https://doi.org/ 10.1007/s11071-014-1753-y
2. Seifried, R.: Integrated mechanical and control design of underactuated multibody systems. Nonlinear Dyn. 67, 1539-1557 (2012). https://doi.org/10.1007/ s11071-011-0087-2

3. Liu, P., Yu, H., Cang, S.: Geometric analysis-based trajectory planning and control for underactuated capsule systems with viscoelastic property. Trans. Inst. Meas. Control. (2017). https://doi.org/10.1177/0142331217708833

4. Zhang, X., Fang, Y., Sun, N.: Minimum-time trajectory planning for underactuated overhead crane systems with state and control constraints. IEEE Trans. Ind. Electron. 61, 69156925 (2014). https://doi.org/10.1109/TIE.2014.2320231

5. Liu, P., Yu, H., Cang, S.: Modelling and dynamic analysis of underactuated capsule systems with friction-induced hysteresis. In: 2016 IEEE/RSJ international conference on intelligent robots and systems (IROS), pp. 549-554. IEEE (2016)

6. Fang, Y., Ma, B., Wang, P., Zhang, X.: A motion planningbased adaptive control method for an underactuated crane system. Control Syst. Technol. IEEE Trans. 20, 241-248 (2012)

7. Nguyen, K.-D., Dankowicz, H.: Adaptive control of underactuated robots with unmodeled dynamics. Robot. Auton. Syst. 64, 84-99 (2015). https://doi.org/10.1016/j.robot. 2014.10.009

8. Liu, P., Yu, H., Cang, S.: On periodically pendulum-driven systems for underactuated locomotion: a viscoelastic jointed model. In: 2015 21st International Conference on Automation and Computing (ICAC). pp. 1-6 (2015)

9. Liu, P., Yu, H., Cang, S.: Modelling and analysis of dynamic frictional interactions of vibro-driven capsule systems with viscoelastic property. Eur. J. Mech.-A Solids. 74, 16-25 (2019)

10. Liu, P., Huda, M.N., Tang, Z., Sun, L.: A self-propelled robotic system with a visco-elastic joint: dynamics and motion analysis. Eng. Comput. (2019). https://doi.org/10. 1007/s00366-019-00722-3

11. Liu, P., Yu, H., Cang, S.: Trajectory synthesis and optimization of an underactuated microrobotic system with dynamic constraints and couplings. Int. J. Control Autom. Syst. 16, 2373-2383 (2018)

12. Liu, P., Yu, H., Cang, S.: Optimized adaptive tracking control for an underactuated vibro-driven capsule system. Nonlinear Dyn. 94, 1803-1817 (2018)

13. Brockett, R.W.: others: Asymptotic stability and feedback stabilization. Differ. Geom. Control Theory. 27, 181-191 (1983)

14. Hwang, C.-L., Chiang, C.-C., Yeh, Y.-W.: Adaptive fuzzy hierarchical sliding-mode control for the trajectory tracking of uncertain underactuated nonlinear dynamic systems. IEEE Trans. Fuzzy Syst. 22, 286-299 (2014)

15. Liu, P., Neumann, G., Fu, Q., Pearson, S., Yu, H.: Energyefficient design and control of a vibro-driven robot. In: 2018 IEEE/RSJ International Conference on Intelligent Robots and Systems (IROS), pp. 1464-1469. IEEE (2018)

16. Liu, P., Yu, H., Cang, S.: Modelling and control of an elastically joint-actuated cart-pole underactuated system. In: 2014 20th International Conference on Automation and Computing (ICAC), pp. 26-31. IEEE (2014) 
17. Valentinis, F., Donaire, A., Perez, T.: Energy-based motion control of a slender hull unmanned underwater vehicle. Ocean Eng. 104, 604-616 (2015)

18. Liu, P.: Bio-inspired robotic control in underactuation: principles for energy efficacy, dynamic compliance interactions and adaptability (2018). https://ethos.bl.uk/OrderDetails. do?uin=uk.bl.ethos.732064

19. Mistry, M., Buchli, J., Schaal, S.: Inverse dynamics control of floating base systems using orthogonal decomposition. In: 2010 IEEE International Conference on Robotics and Automation (ICRA), pp. 3406-3412. IEEE (2010)

20. Blajer, W., Dziewiecki, K., Kołodziejczyk, K., Mazur, Z.: Inverse dynamics of underactuated mechanical systems: a simple case study and experimental verification. Commun. Nonlinear Sci. Numer. Simul. 16, 2265-2272 (2011)

21. Yue, M., An, C., Du, Y., Sun, J.: Indirect adaptive fuzzy control for a nonholonomic/underactuated wheeled inverted pendulum vehicle based on a data-driven trajectory planner. Fuzzy Sets Syst. 290, 158-177 (2016). https://doi.org/10. 1016/j.fss.2015.08.013

22. Xu, J.-X., Guo, Z.-Q., Lee, T.H.: Design and implementation of integral sliding-mode control on an underactuated twowheeled mobile robot. IEEE Trans. Ind. Electron. 61, 36713681 (2014)

23. Xin, X., Tanaka, S., She, J., Yamasaki, T.: New analytical results of energy-based swing-up control for the Pendubot. Int. J. Non-Linear Mech. 52, 110-118 (2013). https://doi. org/10.1016/j.ijnonlinmec.2013.02.003

24. Cornejo, C., Alvarez-Icaza, L.: Passivity based control of under-actuated mechanical systems with nonlinear dynamic friction. J. Vib. Control. (2011). https://doi.org/10.1177/ 1077546311408469

25. Peng, Z., Wang, D., Chen, Z., Hu, X., Lan, W.: Adaptive dynamic surface control for formations of autonomous surface vehicles with uncertain dynamics. IEEE Trans. Control Syst. Technol. 21, 513-520 (2013). https://doi.org/10.1109/ TCST.2011.2181513

26. Cong, S., Liang, Y.: PID-like neural network nonlinear adaptive control for uncertain multivariable motion control systems. IEEE Trans. Ind. Electron. 56, 3872-3879 (2009)

27. Sazonov, E.S., Klinkhachorn, P., Klein, R.L.: Hybrid LQGneural controller for inverted pendulum system. In: Proceedings of the 35th Southeastern Symposium on System Theory, 2003, pp. 206-210. IEEE (2003)

28. Sprangers, O., Babuška, R., Nageshrao, S.P., Lopes, G.A.: Reinforcement learning for port-Hamiltonian systems. IEEE Trans. Cybern. 45, 1017-1027 (2015)

29. Li, J., Guo, X., Li, Z., Chen, W.: Stochastic adaptive optimal control of under-actuated robots using neural networks. Neurocomputing. 142, 190-200 (2014). https://doi.org/10. 1016/j.neucom.2014.04.049

30. Yang, C., Li, Z., Cui, R., Xu, B.: Neural network-based motion control of an underactuated wheeled inverted pendulum model. IEEE Trans. Neural Netw. Learn. Syst. 25, 2004-2016 (2014). https://doi.org/10.1109/TNNLS.2014. 2302475

31. Tong, S.C., Li, Y.M., Zhang, H.G.: Adaptive neural network decentralized backstepping output-feedback control for nonlinear large-scale systems with time delays. IEEE Trans. Neural Netw. 22, 1073-1086 (2011). https://doi.org/ 10.1109/TNN.2011.2146274
32. Mohareri, O., Dhaouadi, R., Rad, A.B.: Indirect adaptive tracking control of a nonholonomic mobile robot via neural networks. Neurocomputing. 88, 54-66 (2012). https://doi. org/10.1016/j.neucom.2011.06.035

33. A biologically inspired approach to tracking control of underactuated surface vessels subject to unknown dynamics-ScienceDirect. http://www.sciencedirect.com/ science/article/pii/S0957417414005958

34. Hsu, C.-F.: Adaptive backstepping Elman-based neural control for unknown nonlinear systems. Neurocomputing. 136, 170-179 (2014)

35. Ping, Z.: Tracking problems of a spherical inverted pendulum via neural network enhanced design. Neurocomputing. 106, 137-147 (2013)

36. Jung, S., Kim, S.S.: Control experiment of a wheel-driven mobile inverted pendulum using neural network. IEEE Trans. Control Syst. Technol. 16, 297-303 (2008). https:// doi.org/10.1109/TCST.2007.903396

37. Liu, D., Wang, D., Zhao, D., Wei, Q., Jin, N.: Neuralnetwork-based optimal control for a class of unknown discrete-time nonlinear systems using globalized dual heuristic programming. IEEE Trans. Autom. Sci. Eng. 9, 628-634 (2012)

38. Liu, Y.-J., Chen, C.P., Wen, G.-X., Tong, S.: Adaptive neural output feedback tracking control for a class of uncertain discrete-time nonlinear systems. IEEE Trans. Neural Netw. 22, 1162-1167 (2011)

39. Xu, B., Sun, F., Yang, C., Gao, D., Ren, J.: Adaptive discretetime controller design with neural network for hypersonic flight vehicle via back-stepping. Int. J. Control 84, 15431552 (2011)

40. Zhang, H., Qin, C., Luo, Y.: Neural-network-based constrained optimal control scheme for discrete-time switched nonlinear system using dual heuristic programming. IEEE Trans. Autom. Sci. Eng. 11, 839-849 (2014)

41. Wang, T., Gao, H., Qiu, J.: A combined adaptive neural network and nonlinear model predictive control for multirate networked industrial process control. IEEE Trans. Neural Netw. Learn. Syst. 27, 416-425 (2016)

42. Zhang, H., Wang, Z., Liu, D.: A comprehensive review of stability analysis of continuous-time recurrent neural networks. IEEE Trans. Neural Netw. Learn. Syst. 25, 12291262 (2014)

43. Zhang, H., Cui, L., Luo, Y.: Near-optimal control for nonzero-sum differential games of continuous-time nonlinear systems using single-network ADP. IEEE Trans. Cybern. 43, 206-216 (2013)

44. Zou, A.-M., Kumar, K.D., Hou, Z.-G., Liu, X.: Finite-time attitude tracking control for spacecraft using terminal sliding mode and Chebyshev neural network. IEEE Trans. Syst. Man Cybern. Part B Cybern. 41, 950-963 (2011)

45. Pucci, D., Romano, F., Nori, F.: Collocated adaptive control of underactuated mechanical systems. IEEE Trans. Robot. 31, 1527-1536 (2015)

46. Yang, C., Li, Z., Li, J.: Trajectory planning and optimized adaptive control for a class of wheeled inverted pendulum vehicle models. Cybern. IEEE Trans. 43, 24-36 (2013)

47. Spong, M.W.: Underactuated mechanical systems. In: Siciliano, B., Valavanis, K.P. (eds.) Control Problems in Robotics and Automation. Lecture Notes in Control and Information 
Sciences, vol. 230, pp. 135-150. Springer, Berlin, Heidelberg (1998)

48. Li, H., Furuta, K., Chernousko, F.L.: Motion generation of the capsubot using internal force and static friction. In: Proceedings of the 45th IEEE Conference on Decision and Control, pp. 6575-6580. IEEE (2006)
49. Yu, H., Liu, Y., Yang, T.: Closed-loop tracking control of a pendulum-driven cart-pole underactuated system. Proc. Inst. Mech. Eng. Part J. Syst. Control Eng. 222, 109-125 (2008)

Publisher's Note Springer Nature remains neutral with regard to jurisdictional claims in published maps and institutional affiliations. 